\title{
Un modelo macroeconómico con agentes de vida finita y estocástica: cobertura de riesgo de mercado con derivados americanos*
}

\author{
Ma. Teresa V. Martínez-Palacios** y Francisco Venegas-Martínez***
}

\section{RESUMEN}

En esta investigación se desarrolló un modelo de economía estocástica, pequeña y abierta, poblada por consumidores racionales idénticos que tienen vida finita, pero estocástica; además, son adversos al riesgo y disponen de una riqueza inicial. Estos agentes enfrentan la decisión de distribuir su riqueza entre consumo e inversión en un portafolio de activos en un ambiente de riesgo de mercado y de política fiscal incierta. La cobertura se lleva a cabo mediante una opción americana de venta y su valuación se realiza en términos de cuánto está dispuesto a pagar el consumidor representativo por mantener dicho contrato a fin de cubrir un activo riesgoso contra caídas en su precio. El precio del contrato se determina en términos del premio al riesgo, el cual se caracteriza mediante la solución de una ecuación diferencial parcial lineal de segundo orden. Por último, se obtiene una formula de aproximación para el precio de la opción americana y se realiza un análisis de sensibilidad de dicho precio con respecto de sus parámetros.

Palabras clave: decisiones de consumo y portafolio, valuación de productos derivados, política fiscal, riesgo de mercado, modelos de equilibrio.

Clasificación JEL: D91, G13, E62, D50, D81.

\begin{abstract}
This paper develops a stochastic model of a small and open economy populated by identical rational consumers having finite life but stochastic, who are risk averse and have an initial wealth. These agents face the decision to allocate his wealth between consumption and investment in a portfolio of assets under an environment of risk market and uncertain fiscal policy. Hedging is performed via an American put option and its pricing is carried out in terms of how much the representative consumer is willing to pay to keep that contract to hedge a fall in the risky asset price. The option price is determined in terms of the risk premium, which is characterized by the solution of a second-order, linear partial differential equation. Finally, an approximated formula for the American option price is obtained, and a sensitive analysis of such a price with respect to its parameters is carried out.
\end{abstract}

Keywords: consumption y portfolio decisions, pricing contingent claims, fiscal policy, market risk, equilibrium models.

JEL classification: D91, G13, E62, D50, D81.

* Fecha de recepción: 27/09/2012. Fecha de aprobación: 11/09/2014.

** Profesora-investigadora de tiempo completo en la Escuela Superior de Apan de la Universidad Autónoma del Estado de Hidalgo. Correo electrónico: terevioleta@hotmail.com.

*** Profesor-investigador de tiempo completo definitivo, de la Escuela Superior de Economía del Instituto Politécnico Nacional. Correo electrónico: fvenegas1111@yahoo.com.mx. 


\section{INTRODUCCIÓN}

En un ambiente de incertidumbre económica y financiera como el que muestra la realidad contingente actual, con cambios constantes de política económica $\mathrm{y}$ volatilidad intermitente en los mercados financieros, las decisiones sobre consumo, portafolio y producción de los agentes económicos son afectadas. La necesidad de los diversos agentes que participan en la economía de identificar los patrones que caracterizan estos cambios para tomar decisiones oportunas acordes con el contexto es un tema que, sin duda, ha recobrado vigencia y relevancia actual.

A este respecto, existen diversos modelos disponibles en la literatura en los cuales se supone que las variables económicas y financieras relevantes son conducidas por procesos estocásticos de diferente naturaleza, por ejemplo, procesos de difusión o de difusión con saltos, como se observa en Merton (1971; 1992), Turnovsky (1993), Hernández-Lerma (1994), Venegas-Martínez (2001; 2006) y Turnovsky y Smith (2006). Otro aspecto que es de igual relevancia es el desarrollo de modelos macroeconómicos estocásticos que expliquen hechos estilizados en las decisiones de consumo y portafolio de los agentes; véase, al respecto, Grinols y Turnovsky (1993); Venegas-Martínez (2004; 2005); Turnovsky y Smith (2006), y Venegas-Martínez, Ortiz-Arango y Castillo-Ramírez (2010), entre otros.

Una particularidad de los modelos estocásticos que da coherencia a la realidad contingente son las funciones de tiempo de paro; sobre este temas, se puede consultar a Bjork, Myhrman y Persson (1987); Karatzas y Shreve (1988); Merton (1992); Hernández-Lerma (1994); Shreve (1997); Kohn (2003); Bjork (2004); Villeneuve (2007), y Kohn ${ }^{1}$ (2011), entre otros. Por ejemplo, Shreve (1997) analiza el tiempo de ejercicio óptimo de opción de venta americana realizando su valuación mediante un modelo binomial en el que el tiempo de paro correspondiente se define como primer instante en que la opción es igual a su valor intrínseco. Así mismo, Hernández-Lerma (1994) aplica el concepto de tiempo de paro como un tiempo de salida óptima para procesos de difusión controlados. Por su parte, Bjork, Myhrman Persson (1987) y Björk (2004) imponen un tiempo de paro para no generar soluciones de control degeneradas.

\footnotetext{
${ }^{1}$ Es oportuno mencionar que, a excepción de Kohn, los autores citados aplican tiempos de paro en conjunto mediante la clase de modelos económico-matemáticos llamados de equilibrio general dinámico y estocástico (EGDE).
} 
Dos de los temas distintivos, en el marco de los modelos anteriores, son la obtención de precios de activos financieros y la valuación de productos derivados, cuya literatura es muy vasta y variada; considérense al respecto los artículos, algunos seminales y otros de referencia, de Black y Scholes (1973); Merton (1973); Cox y Ross (1976); Cox, Ingersoll y Ross (1985a; 1985b); Geske y Shastri (1985); Ho y Lee (1986); Hull y White (1987; 1993); Detemple y Tian (2002); Venegas-Martínez (2006; 2007; 2010); Sierra (2007); ${ }^{2}$ Ángeles-Castro y Venegas-Martínez (2010), y Venegas-Martínez y Cruz-Ake (2010), entre otros. Una característica común, tal vez una limitación, que guardan estas investigaciones es que consideran una temporalidad, infinita o finita, determinista para la valuación de derivados.

Las opciones americanas, a diferencia de las opciones europeas, pueden ser ejercidas en cualquier momento entre el día en que se pactan y el día de vencimiento, ambos inclusive, y se negocian en mercados organizados y reconocidos por las autoridades financieras (bolsas de opciones) o mercados extrabusátiles (отс, por sus siglas en inglés: over the counter) o informales. No obstante, es importante señalar que la mayor parte de las opciones que se operan en estos mercados son justamente del tipo americano. La investigación que se ha realizado en este tema es también muy extensa; véase, por ejemplo, Taylor (1967); Merton (1973); Cox, Ross y Rubinstein (1979); Whaley (1981); Barone-Adesi y Whaley (1987); Broadie y Detemple (1996; 2004); Huyen (1997); Ju (1998); Broadie, Glasserman y Ha (2000); Fu et al. (2001); Duan y Simonato (2001); Longstaff y Schwartz (2001); Clement, Lamberton y Protter (2002); Detemple y Tian (2002); Rogers (2002); Villeneuve y Zanette (2002); Detemple, Feng y Tian (2003); Kou y Wang (2004); Stentoft (2005); Ikonen y Toivanen (2008), y Kohn (2011), entre otros.

Como resultado de la temporalidad anticipada de una opción americana, parte del problema de valuación consiste en identificar la frontera de ejercicio que maximice el valor de la opción para el dueño del contrato; al respecto, véase Merton (1973) y Taylor (1967). A pesar de que lo más conveniente es disponer de una fórmula cerrada, no se cuenta con ella en este caso, por lo que los esfuerzos en investigación se han concentrado en el desarrollo de métodos aproximados. Así, por ejemplo, se encuentran entre los trabajos que utilizan métodos

\footnotetext{
${ }^{2}$ Una particularidad del trabajo de este autor es que sus activos son modelados mediante movimientos brownianos fraccionales.
} 
numéricos los de Villeneuve y Zanette (2002) e Ikonen y Toivanen (2008)3; el método de diferencias finitas, el de Brennan y Schwartz (1977), y el método de árbol binomial, el de Cox, Ross y Rubinstein (1979). Por su parte, Whaley (1981) y Barone-Adesi y Whaley (1987) desarrollan una fórmula analítica aproximada que es muy rápida en comparación con otros métodos; así mismo, Kou y Wang (2004) demuestran que mediante un modelo de difusión con saltos se puede llegar a una aproximación analítica cuando el horizonte es finito. Algunos otros investigadores adoptan un esquema de interpolación para el precio de la opción americana, como Broadie y Detemple (1996), Villeneuve y Zanette (2002) y Detemple, Feng y Tian (2003). Dentro de los métodos $^{4}$ que son esquemas de aproximación de frontera libre se encuentra el de representación integral; como ejemplo se tienen las investigaciones de Broadie y Detemple (1996) y de Ju (1998). Una característica común entre estos métodos es el uso de tiempos de paro para la valuación de los derivados americanos; véase al respecto Merton (1990); Karatzas y Shreve (1988); Villeneuve y Zanette (2002); Detemple, Feng y Tian (2003); Bjork (2004); Villeneuve (2007), y Kohn (2011), ${ }^{6}$ entre otros.

En este trabajo se desarrolla un modelo de una economía pequeña y abierta poblada por consumidores racionales, maximizadores de utilidad y adversos al riesgo, que disponen de una riqueza inicial y enfrentan la decisión de distribuir su riqueza entre consumo e inversión en un portafolio cuando el horizonte de planeación del que disponen es finito con fecha final estocástica. En este modelo, los agentes se desenvuelven en un ambiente de riesgo de mercado y de política fiscal incierta. La valuación de la opción de venta americana es llevada a cabo en términos de cuánto está dispuesto a pagar un consumidor representativo por mantener un contrato de este tipo a fin de cubrir un activo riesgoso contra el riesgo de mercado al mismo tiempo que maximiza su utilidad por el consumo. Las características distintivas de este trabajo, en el marco de las investigaciones anteriormente mencionadas, son: 1) la economía es pequeña y abierta;

\footnotetext{
${ }^{3}$ En realidad Ikonen y Toivanen (2008) describen y comparan cinco métodos numéricos para el precio de opciones americanas de venta bajo el modelo de volatilidad estocástica de Heston (1993).

${ }^{4}$ Algunos de estos métodos se deben a Geske y Johnson (1984) y Huang, Subrahmanyam y Yu (1996), entre otros.

${ }^{5}$ El método integral que utilizan Broadie y Detemple (1996), así como Ju (1998), involucra solamente la función normal acumulativa univariada, lo que lo hace muy rápido, aunque no muy preciso.

${ }^{6}$ Kohn (2011) examina el problema del paro óptimo para maximizar el valor presente esperado en el ejercicio de una opción de venta americana con tiempo de madurez perpetuo y también para el caso finito, sobre todos los posibles tiempos de ejercicio.
} 
2) el horizonte de planeación es finito, de fecha final estocástica; 3) la valuación del producto derivado americano se efectúa con base en la racionalidad económica y una tasa de interés no sólo libre de riesgo de incumplimiento, sino también de riesgo inflacionario; 4) el diseño del proceso de optimización evita que el inversor caiga en bancarrota; ${ }^{7} 5$ ) el precio se caracteriza en términos del premio al riesgo, el cual se calcula como la solución de una ecuación diferencial parcial lineal de segundo orden.

Este trabajo consta de ocho partes, además de la introducción y las conclusiones. En la primera, se describe el marco teórico de la investigación. En el segundo apartado, se establece la restricción presupuestal intertemporal del agente representativo de una economía con incertidumbre. La tercera sección introduce el horizonte de planeación finito y de magnitud estocástica a través de un problema de control en tiempo continuo de consumo e inversión óptimos en un ambiente de riesgo de mercado y de política fiscal incierta. En la restricción presupuestal se incluye la tenencia de una opción americana de venta con un subyacente conducido por el movimiento geométrico browniano.

En la cuarta parte se proporciona una solución al problema de control óptimo planteado, mediante la cual se obtienen como resultados centrales la ecuación diferencial parcial de Hamilton-Jacobi-Bellman y las condiciones de primer orden que llevan a las decisiones óptimas de las variables de control. En la quinta sección se obtiene el premio al riesgo de mercado de una opción americana de venta. Mientras tanto, en la sexta, se establece una caracterización del precio de una opción americana de venta en términos del premio al riesgo de mercado, el cual se obtiene mediante la solución de una ecuación diferencial parcial lineal de segundo orden. En la séptima sección se logra una aproximación del precio de una opción americana de venta y se realiza un análisis de sensibilidad con respecto de sus parámetros. A su vez, en la octava, sección se lleva a cabo la verificación de la solución encontrada con programación dinámica del modelo. Por último, se presentan las conclusiones destacando las limitaciones e indicando aquellas extensiones que serán tomadas en cuenta en la agenda futura de investigación.

\footnotetext{
${ }^{7}$ En realidad, el argumento que aquí se utiliza para evitar la bancarrota, interpretada como la insolvencia del agente económico, no cumple en el sentido amplio con el análisis realizado por Sethi (1997). El argumento de tiempo de paro en conjunción con la condición de frontera (véase al respecto Hernandez-Lerma (1994) y Bjork (2004)) impuesta a la ecuación de HJB y el uso de una función de utilidad de clase HARA impiden que el problema degenere y, por ende, financieramente evite la bancarrota del agente económico representativo.
} 


\section{MARCo TeÓRICO}

A través del equilibrio general dinámico estocástico, se analiza un modelo de un consumidor racional representativo ${ }^{8}$ que se desenvuelve en una economía pequeña y abierta, en la que se produce y se consume un solo bien de carácter perecedero, que es internacionalmente comercializable.

\section{Activos y rendimientos}

Se considera un consumidor que es al mismo tiempo productor y un intervalo de tiempo fijo $[0, T]$ con $T$ como una variable aleatoria que se definirá más adelante. En el tiempo $t=0$ el consumidor es dotado con una riqueza inicial $X_{0}$ y el problema que enfrenta es cómo distribuir su riqueza entre consumo e inversión en un portafolio, de tal forma que su riqueza se mantenga positiva sobre el horizonte de planeación y tal que maximice su utilidad total esperada y descontada. Específicamente, se supone que la utilidad del agente está dada por

$$
E\left[\int_{0}^{T} F\left(t, c_{t}\right) d t+\Phi\left(X_{T}\right) \mid F_{0}\right]
$$

en donde $F$ es la satisfacción (descontada) para consumo y $\Phi$ es la función de legado o herencia (o función de retiro) al tiempo $T$, la cual mide la utilidad de contar con recursos al final del período y $F_{0}$ es la información relevante al tiempo $t=0$.

Para maximizar dicha utilidad, se permite que el agente asigne sus recursos a consumo e inversión en activos con y sin riesgo: dinero (nominal) doméstico $M_{t}$, bonos gubernamentales (nominales) domésticos $B_{t}$, acciones domésticas $k_{t}$, un contrato doméstico de opción americana de venta $V_{t}$ y bonos (reales) extranjeros $b_{t}^{*}$. De tal forma que la riqueza real total, $x_{t}$, está dada por

$$
x_{t}=m_{t}+b_{t}+k_{t}+V_{t}+b_{t}^{*}
$$

donde $M_{t} / P_{t}=m_{t}$ son los saldos monetarios reales y $B_{t} / P_{t}=b_{t}$ es un bono gubernamental doméstico en términos reales.

\footnotetext{
${ }^{8}$ Véase Ramsey (1928).
} 


\section{Dinámica del nivel general de precios}

Se supone que en esta economía el nivel general de precios domésticos $P_{t}$ es determinado por la condición de poder de paridad de compra, $P_{t}=e_{t} P_{t}^{*}$, donde $P_{t}^{*}$ es el precio en moneda extranjera del bien en el resto del mundo y $e_{t}$ es el tipo de cambio nominal. A efectos de simplificar el modelo, se supondrá, sin pérdida de generalidad, que el tipo de cambio nominal es "relativamente" estable, de tal manera que se puede escribir $e_{t}=c t e$. Por otro lado, como la economía es pequeña, ésta es precio aceptante de $P_{t}^{*}$ y de la misma manera, sin restar generalidad, se supondrá $P_{t}^{*} \equiv 1$.

Así pues, se supone que el agente representativo percibe que la tasa de inflación esperada $d P_{t} / P_{t}$ es conducida por el proceso markoviano de difusión:

$$
\frac{d P_{t}}{P_{t}}=\pi d t+\sigma_{P} d W_{P, t}
$$

donde $\pi$ es la tasa media esperada de inflación, $\sigma_{P}$ es la variación esperada de la tasa de inflación y $W_{P, t}$ es un proceso de Wiener, también llamado movimiento browniano, que está definido sobre un espacio fijo de probabilidad con su filtración aumentada $\left(\Omega, F,\left(F_{t}^{W}\right)_{t \in[0, T]}, P\right)$. En este caso, los parámetros $\pi$ y $\sigma_{P}$ son exógenos.

\section{Saldos monetarios reales}

El agente económico mantiene saldos monetarios reales $M_{t} / P_{t}=m_{t}$, donde $M_{t}$ es el acervo nominal de dinero. La tasa de retorno estocástica por la tenencia de saldos monetarios reales está dada por el cambio porcentual del precio del dinero en términos de los bienes. Se supone además que el rendimiento marginal del dinero es cero, es decir, $d M_{t}=0$. La aplicación del lema de Itô al cambio porcentual del inverso del nivel de precios cuando se considera al nivel general de éstos como el proceso subyacente, conduce al rendimiento por la tenencia de saldos reales

$$
d R_{m, t}=\frac{d\left(M_{t} / P_{t}\right)}{\left(M_{t} / P_{t}\right)}=r_{m} d t-\sigma_{P} d W_{P t},
$$

con $r_{m}=-\pi+\sigma_{P}^{2}$. 


\section{Bono cupón cero}

El agente económico destina una parte de su riqueza a la inversión en un bono gubernamental cupón cero $B_{t}$. Se supone que la tasa nominal de rendimiento que pagan es $i$, es decir, $d B_{t}=B_{t} i\left(l-\tau_{y}\right) d t$, con $\tau_{y}$ el impuesto aplicado a la tasa de interés nominal de un bono gubernamental. El rendimiento por la tenencia de bonos gubernamentales está dado por la siguiente ecuación:

$$
d R_{b, t}=r_{b} d t-\sigma_{P} d W_{P, t}
$$

donde $r_{b}=i\left(1-\tau_{y}\right)-\pi+\sigma_{P}^{2}$. Adicionalmente se supone que $i\left(1-\tau_{y}\right)-\pi>\sigma_{P}^{2}$, para asegurar que el rendimiento se mantenga positivo.

\section{Acciones}

Se supone que los consumidores también son productores y la empresa representativa tiene una función de producción $y=A k_{t}$ que ofrece títulos de capital de acuerdo con el criterio (determinista) de maximización de su beneficio neto $\Pi_{t}=A k_{t}-r_{b} k_{t}$ donde $r_{b}$ es la tasa de interés que pagan los bonos gubernamentales. Una condición necesaria de optimidad del problema anterior es $r_{b}=A$. Así, en lo que sigue, de manera indistinta se puede escribir $r_{b}$ o $A$, lo que determina el equilibrio con el sector productivo.

Otra parte de su riqueza la invierte en una acción, en donde la tasa de rendimiento de las acciones sigue una ecuación diferencial estocástica:

$$
d R_{k} \equiv \frac{d k_{t}}{k_{t}}=r_{k} d t+\sigma_{k} d W_{k t}
$$

donde, $r_{k} \in \mathbf{R}$ es el rendimiento medio esperado y $\sigma_{k}>0$ es la volatilidad instantánea; como antes, $W_{k, t}$ es un proceso de Wiener, o movimiento browniano, definido sobre un espacio de probabilidad adecuado.

\section{Opción americana de venta}

Con el propósito de cubrir su inversión en la acción, el agente también invierte en un contrato de opción americana de venta sobre dicha acción, que tiene precio $V_{t}\left(k_{t}, t\right)$. En otras palabras, si el precio de la acción cae por debajo de un umbral predeterminado, entonces el agente tiene el derecho de vender la acción al valor 
del umbral. El rendimiento de la opción está dado por el cambio porcentual de la prima, es decir,

$$
d R_{V} \equiv \frac{d V_{t}}{V_{t}}
$$

en donde $d V_{t}$ se obtiene mediante el lema de Itô de la siguiente manera:

$$
d V_{t}=\left(\frac{\partial V_{t}}{\partial t}+\frac{\partial V_{t}}{\partial k_{t}} r_{k} k_{t}+\frac{1}{2} \frac{\partial^{2} V_{t}}{\partial k_{t}^{2}} \sigma_{k}^{2} k_{t}^{2}\right) d t+\frac{\partial V_{t}}{\partial k_{t}} \sigma_{k} k_{t} d W_{k t}
$$

Si se denotan

$$
r_{V}=\frac{1}{V_{t}}\left(\frac{\partial V_{t}}{\partial t}+\frac{\partial V_{t}}{\partial k_{t}} r_{k} k_{t}+\frac{1}{2} \frac{\partial^{2} V_{t}}{\partial k_{t}^{2}} \sigma_{k}^{2} k_{t}^{2}\right) \quad \text { y } \quad \sigma_{V}=\frac{1}{V_{t}}\left(\frac{\partial V_{t}}{\partial k_{t}} \sigma_{k} k_{t}\right)
$$

se sigue que

$$
d R_{V} \equiv \frac{d V_{t}}{V_{t}}=r_{V} d t+\sigma_{V} d W_{k t}
$$

y $W_{k, t}$ es tal como se definió en el párrafo anterior y es claro que comparten esta característica de riesgo toda vez que la opción hereda el riesgo de la acción.

\section{Bonos internacionales}

El agente representativo también tiene acceso a un bono internacional, el cual paga una tasa de interés real $r>0$, libre de riesgo de incumplimiento y constante en todos los plazos. Por ello, el agente destina una parte de su riqueza a la inversión, en el tiempo $t$, en un bono que satisface

$$
d b_{t}^{*}=r b_{t}^{*} d t, \text { con } b_{0}^{*} \text { dado, }
$$

lo cual implica que el rendimiento de dicho bono está dado por

$$
d R_{b_{t}^{*}, t} \equiv \frac{d b_{t}^{*}}{b_{t}^{*}}=r_{b}^{*} d t
$$

Con el supuesto de que la economía es pequeña, es decir, tomadora de precios, se asume en particular que es tomadora de la tasa de interés. En otras palabras, la economía doméstica toma $r_{b}{ }^{*}$ como la tasa de interés real (libre de riesgo de incumplimiento). En consecuencia, $r_{b}{ }^{*}$ será utilizada para la valua- 
ción de la opción americana de venta. Si consideramos que la economía estadounidense representa al resto del mundo, sin duda la probabilidad de incumplimiento de los bonos del Tesoro es menor que la de los Cetes; por lo tanto, la tasa de interés real estadounidense es un mejor candidato como tasa libre de riesgo (de incumplimiento). Es de observar que en la economía doméstica la tasa real $r_{b}$ se calcula en términos del diferencial de la tasa de interés nominal y la tasa de inflación, y que en esta última hay factores de riesgo que la afectan, como el nivel general de precios. Es decir, $r_{b}$ no necesariamente es una tasa de interés libre de todo riesgo.

\section{Impuesto sobre la riqueza}

Se considera un movimiento browniano $\left(U_{t}\right)_{t>0}$ definido en un espacio de probabilidad fijo con su filtración aumentada $\left(\Omega, F,\left(F_{t}{ }^{U}\right)_{t \in[0, T]}, P\right)$. Se supone que el agente económico prevé que su riqueza será gravada a una tasa incierta $\tau_{t}$ de acuerdo con la siguiente ecuación diferencial estocástica:

$$
\frac{d \tau_{t}}{\tau_{t}}=\bar{\tau} d t+\sigma_{\tau} d \tilde{W_{t}}, \quad \tau_{0}>0
$$

con

$$
\tilde{W}_{t}=\rho W_{P, t}+\sqrt{1-\rho^{2}} U_{t}
$$

y

$$
\operatorname{cov}\left(d W_{P, t}, d\left(\rho W_{P, t}+\sqrt{1-\rho^{2}} U_{t}\right)\right)=\rho d t
$$

donde $\bar{\tau}_{t}$ es la tasa media esperada de crecimiento del impuesto sobre la riqueza, $\sigma_{\tau}$ es la volatilidad de la tasa impositiva sobre la riqueza y $-1 \leq \rho \leq 1$ es la correlación entre los cambios en la inflación y los cambios en el impuesto sobre la riqueza. Se supone que los procesos $W_{P, t}, U_{t}$ y $W_{k, t}$ son independientes dos a dos.

\section{Restricción del tipo cash-in-advance}

Se supone, además, que el consumidor demanda dinero para realizar sus transacciones, lo cual se hace por medio de la restricción cash in advance propuesta por Clower (1967): ${ }^{9}$

\footnotetext{
${ }^{9}$ En palabras de Clower: "goods buy money and money buys goods, but goods don t buy goods".
} 


$$
m_{t}=\alpha c_{t}
$$

En esta restricción, $c_{t}$ es el consumo y $\alpha>0$ es el tiempo que se mantiene el dinero para financiar el consumo. Esta restricción debe cumplirse en todo momento, ya que determina el conjunto factible de la variable de control $c_{t}$.

\section{RESTRICCIÓN PRESUPUESTAL INTERTEMPORAL DEL AGENTE REPRESENTATIVO}

Las proporciones de riqueza que se destinarán a los activos riesgosos, saldos monetarios reales, bonos cupón cero gubernamentales, acción doméstica, la opción americana de venta y el activo sin riesgo, el bono extranjero, en el portafolio de inversión, al tiempo $t$, se denotarán mediante $\theta_{1 t}, \theta_{2 t}, \theta_{3 t}, \theta_{4 t}$, y $1-\theta_{1 t}-\theta_{2 t}-\theta_{3 t}$ $-\theta_{4 t}=1-\sum_{i=1}^{4} \theta_{i t}$, respectivamente; donde $\theta_{i t}=\frac{j_{t}}{X_{t}}$ es la proporción de la riqueza que se destina al activo $j, j=m, b, k, V, b^{*}$. En lo que sigue, $c_{t}$ denota la tasa de consumo, a la que se impone la condición $c_{t} \geq 0, \forall t \geq 0$.

Adicionalmente, se supone que todas las estrategias de consumo e inversión son autofinanciables y que, además, las negociaciones se llevan a cabo en forma continua (los mercados nunca cierran), sin incurrir en ningún momento en costos por comisiones a agentes de casas de bolsa. También, que están permitidas las ventas en corto (pedir acciones prestadas a agentes de bolsa). ${ }^{10}$

De esta manera, la dinámica del proceso de la riqueza del agente está dada por el sistema de ecuaciones diferenciales estocásticas siguiente:

$$
\left\{\begin{aligned}
d X_{t}= & X_{t} \theta_{1 t} d R_{m, t}+X_{t} \theta_{2 t} d R_{b, t}+X_{t} \theta_{3 t} d R_{k, t}+X_{t} \theta_{4 t} d R_{V, t}+ \\
& +X_{t}\left(1-\sum_{i=1}^{4} \theta_{i t}\right) d R_{b_{t}^{*}, t}-X_{t} \tau_{t} d t-\left(1+\tau_{c}\right) c_{t} d t, \\
x_{0}= & m_{0}+b_{0}+k_{0}+V_{0}+b_{0}^{*}>0 \\
d \tau_{t}= & \bar{\tau}_{t} \tau_{t} d t+\sigma_{\tau} \tau_{t} d W_{t}, \quad \tau_{0}>0
\end{aligned}\right.
$$

donde $\tau_{c}$ es una tasa impositiva ad valorem del consumo. Al sustituir las ecuaciones (3) a (10) en el sistema de ecuaciones (13), se tiene que el proceso de la riqueza está dado por el siguiente sistema de ecuaciones diferenciales:

\footnotetext{
${ }^{10}$ Por supuesto que hasta donde el tiempo de paro lo permita.
} 
82 ECONOMÍA: TEORÍA Y PRÁCTICA • Nueva Época, número 41, julio-diciembre 2014

$$
\left\{\begin{array}{l}
d X_{t}=\left\lfloor X_{t} \theta_{1 t} r_{m}+X_{t} \theta_{2 t} r_{b}+X_{t} \theta_{3 t} r_{k}+X_{t} \theta_{4 t} r_{V}+X_{t}\left(1-\sum_{i=1}^{4} \theta_{i t}\right) r_{b}^{*}-X_{t} \tau_{t}-\left(1+\tau_{c}\right) c_{t}\right\rfloor d t \\
-X_{t}\left(\theta_{1 t}+\theta_{2 t}\right) \sigma_{p} d W_{P, t}+X_{t} \theta_{3 t} \sigma_{k} \mathrm{~d} W_{k, t}+X_{t} \theta_{4 t} \sigma_{V} d W_{k, t} \quad x_{0}=m_{0}+b_{0}+k_{0}+V_{0}+b_{0}^{*}>0 \\
d \tau_{t}=\bar{\tau}_{t} \tau_{t} d t+\sigma_{\tau} \tau_{t}\left(\rho d W_{P, t}+\sqrt{1-\rho^{2}} d U_{t}\right), \quad \tau_{0}>0
\end{array}\right.
$$

equivalentemente

$$
\left\{\begin{array}{l}
d X_{t}=X_{t} r_{x} d t+X_{t} \sigma_{p, t} d W_{P, t}+X_{t} \sigma_{k, t} d W_{k, t}, \quad x_{0}=m_{0}+b_{0}+k_{0}+V_{0}+b_{0}^{*}>0, \\
d \tau_{t}=\bar{\tau}_{t} \tau_{t} d t+\sigma_{\tau} \tau_{t}\left(\rho d W_{P, t}+\sqrt{1-\rho^{2}} d U_{t}\right), \quad \tau_{0}>0
\end{array}\right.
$$

donde

$$
\begin{gathered}
r_{x}=\theta_{1 t} r_{m}+\theta_{2 t} r_{b}+\theta_{3 t} r_{k}+\theta_{4 t} r_{V}+\left(1-\sum_{i=1}^{4} \theta_{i t}\right) r_{b}^{*}-\tau_{t}-\left(1+\tau_{c}\right) \frac{c_{t}}{X_{t}} \\
\sigma_{p, t}=\left(-\theta_{1 t}-\theta_{2 t}\right) \sigma_{p} \quad \text { y } \quad \sigma_{k, t}=\left(\theta_{3 t} \sigma_{k}+\theta_{4 t} \sigma_{V}\right) .
\end{gathered}
$$

Al introducir la restricción cash in advance se tiene $c_{t}=\theta_{1 t} X_{t} \alpha^{-1}$, de donde (16) queda dada por

$$
r_{x}=\theta_{1 t} r_{m}+\theta_{2 t} r_{b}+\theta_{3 t} r_{k}+\theta_{4 t} r_{V}+\left(1-\sum_{i=1}^{4} \theta_{i t}\right) r_{b}^{*}-\tau_{t}-\left(1+\tau_{c}\right) \frac{\theta_{1 t}}{\alpha}
$$

con

$$
\sigma_{p, t}=\left(-\theta_{1 t}-\theta_{2 t}\right) \sigma_{p} \quad \text { y } \quad \sigma_{k, t}=\left(\theta_{3 t} \sigma_{k}+\theta_{4 t} \sigma_{V}\right) .
$$

\section{Problema de ConTrol ÓPTIMo estocástico}

A continuación se establece el problema de control óptimo estocástico que debe resolver el consumidor-inversionista representativo.

\section{Tiempo de paro}

Para estar seguros de no formular un problema degenerado y sea posible para el agente representativo alcanzar el óptimo de su portafolio de consumo e inversión, se restringirá el dominio (horizonte temporal) a $D=[0, T] \times\{x \mid x>0\}$, y se define la función tiempo de paro $\varphi$, dada por 


$$
\varphi=\min \left[\inf \left\{t>0 \mid X_{t}=0\right\}, T\right]
$$

lo que formula al horizonte temporal finito como una variable aleatoria. La interpretación correspondiente es que cuando el proceso de riqueza alcance la frontera del dominio se vuelva cero; entonces, la actividad se termina y ya no hay herencia, de manera que lo natural es que $\Phi$ sea cero.

\section{Problema de control óptimo estocástico}

Al establecer formalmente el problema de la maximización de la utilidad del consumidor como un problema de control óptimo estocástico, se tiene

$$
\begin{aligned}
& \underset{\theta_{1 t}, \theta_{2 t}, \theta_{3}, \theta_{t}, c_{t}}{\operatorname{aximizar}} E\left[\int_{0}^{\varphi} F\left(t, c_{t}\right) d t \mid F_{0}\right], \\
& \left\{\begin{array}{l}
d X_{t}=X_{t} r_{x} d t+X_{t} \sigma_{p, t} d W_{P, t}+X_{t} \sigma_{k, t} d W_{k, t}, \quad x_{0}=m_{0}+b_{0}+k_{0}+V_{0}+b_{0}^{*}>0, \\
d \tau_{t}=\bar{\tau}_{t} \tau_{t} d t+\sigma_{\tau} \tau_{t} d\left(\rho d W_{P, t}+\sqrt{1-\rho^{2}} d U_{t}\right), \quad \tau_{0}>0,
\end{array}\right. \\
& X_{0}=x_{0}, \\
& c_{t} \geq 0, \forall t \geq 0 .
\end{aligned}
$$

\section{Programación dinámica: eCuación diferencial ParCiaL DE HAMilton-JaCOBi-BELlMAN}

Para dar solución al problema (19) y encontrar las proporciones óptimas en el portafolio de inversión y el consumo del agente maximizador, definimos la función de valor del problema mediante la siguiente expresión:

$$
\begin{aligned}
& J\left(X, \tau_{t}, t\right)=\max _{\theta_{t}, \theta_{2 t}, \theta_{3 t}, \theta_{t t} \in \mathbf{R}, 0 \leq\left. c_{s}\right|_{t, t, q]}} E\left[\int_{t}^{\varphi} F\left(c_{s}, s\right) d s \mid F_{t}\right] \\
& =\max _{\theta_{1 t}, \theta_{2 t}, \theta_{3,}, \theta_{t} \in \mathbf{R}, 0 \leq\left. c_{s}\right|_{t, q, q]}} E\left[\int_{t}^{t+d t} F\left(c_{s}, s\right) d s+\int_{t+d t}^{\varphi} F\left(c_{s}, s\right) d s \mid F_{t}\right] .
\end{aligned}
$$

Después de aplicar al primer sumando el teorema del valor medio del cálculo integral y recursividad al segundo sumando, se obtiene que 
84 ECONOMÍA: TEORÍA Y PRÁCTICA • Nueva Época, número 41, julio-diciembre 2014

$$
J\left(X_{t}, \tau_{t}, t\right)=\max _{\theta_{1 t}, \theta_{2 t}, \theta_{3 t}, \theta_{4 t} \in \mathbf{R}, 0 \leq\left. c_{s}\right|_{[t, t+d t]}}\left\{F\left(c_{t}, t\right) d t+o(d t)+J\left(X_{t}+d X_{t}, \tau_{t}+d \tau_{t}, t+d t\right) F_{t}\right\}
$$

Al emplear la expansión en serie de Taylor en el segundo sumando, se tiene

$$
J\left(X_{t}, \tau_{t}, t\right)=\max _{\theta_{1 t}, \theta_{2 t}, \theta_{3 t}, \theta_{4 t} \in \mathbf{R}, 0 \leq\left. c_{s}\right|_{[t, t+d t]}}\left\{F\left(c_{t}, t\right) d t+o(d t)+J\left(X_{t}, \tau_{t}, t\right)+d J\left(X_{t}, \tau_{t}, t\right)+o(d t) \mid F_{t}\right\},
$$

consecuentemente

$$
0=\max _{\theta_{1 t}, \theta_{2 t}, \theta_{3 t}, \theta_{4 t} \in \mathbf{R}, 0 \leq\left. c_{s}\right|_{[t, t+d t]}}\left\{F\left(c_{t}, t\right) d t+o(d t)+d J\left(X_{t}, \tau_{t}, t\right) \mid F_{t}\right\} .
$$

Al aplicar el lema de Itô a $\left.d J\left(X_{t}, \tau_{t}, t\right)\right)$ y simplificar, se obtiene

$$
\begin{aligned}
& 0=\max _{\theta_{1 t}, \theta_{2 t}, \theta_{3 t}, \theta_{4 t} \in \mathbf{R}, 0 \leq\left. c_{s}\right|_{[t, t+d t]}}\left\{F\left(c_{t}, t\right) d t+o(d t)+\frac{\partial J\left(X_{t}, \tau_{t}, t\right)}{\partial X_{t}}\left[X_{t} \sigma_{P, t} d W_{P, t}+X_{t} \sigma_{k, t} d W_{k, t}\right]+\right. \\
& \frac{\partial J\left(X_{t}, \tau_{t}, t\right)}{\partial \tau_{t}}\left[\tau_{t} \sigma_{\tau} \rho d W_{P, t}+\tau_{t} \sigma_{\tau}\left(\sqrt{1-\rho^{2}}\right) d U_{t}\right]+\left[\frac{\partial J\left(X_{t}, \tau_{t}, t\right)}{\partial t}+\frac{\partial J\left(X_{t}, \tau_{t}, t\right)}{\partial X_{t}} X_{t} r_{x}+\right. \\
& \frac{\partial J\left(X_{t}, \tau_{t}, t\right)}{\partial \tau_{t}} \tau_{t} \bar{\tau}_{t}+\frac{1}{2}\left[\frac{\partial^{2} J\left(X_{t}, \tau_{t}, t\right)}{\partial X_{t}^{2}}\left(X_{t}^{2} \sigma_{P, t}^{2}+X_{t}^{2} \sigma_{k, t}^{2}\right)+2 \frac{\partial^{2} J\left(X_{t}, \tau_{t}, t\right)}{\partial X_{t} \partial \tau_{t}}\left(X_{t} \tau_{t} \sigma_{\tau} \rho \sigma_{P, t}\right)+\right. \\
& \left.\left.\left.\frac{\partial^{2} J\left(X_{t}, \tau_{t}, t\right)}{\partial \tau_{t}^{2}}\left(\tau_{t}^{2} \sigma_{\tau}^{2} \rho^{2}+\tau_{t}^{2} \sigma_{\tau}^{2}\left(1-\rho^{2}\right)\right)\right]\right] d t \mid F_{t}\right\} .
\end{aligned}
$$

A continuación se encuentra el valor esperado de esta última ecuación y, dado que $d W_{P, t}, d W_{k, t}, y d U_{t}$ se distribuyen $N(0, d t)$, se eliminarán los términos con movimientos brownianos, de lo que resulta

$$
\begin{aligned}
& 0=\max _{\theta_{1 t}, \theta_{2 t}, \theta_{3 t}, \theta_{4 t} \in \mathbf{R}, 0 \leq\left. c_{s}\right|_{[t, t+t]}}\left\{F\left(c_{t}, t\right) d t+o(d t)+\left[\frac{\partial J\left(X_{t}, \tau_{t}, t\right)}{\partial t}+\frac{\partial J\left(X_{t}, \tau_{t}, t\right)}{\partial X_{t}} X_{t} r_{x}+\right.\right. \\
& \frac{\partial J\left(X_{t}, \tau_{t}, t\right)}{\partial \tau_{t}} \tau_{t} \bar{\tau}_{t}+\frac{1}{2}\left[\frac{\partial^{2} J\left(X_{t}, \tau_{t}, t\right)}{\partial X_{t}^{2}}\left(X_{t}^{2} \sigma_{P, t}^{2}+X_{t}^{2} \sigma_{k, t}^{2}\right)+2 \frac{\partial^{2} J\left(X_{t}, \tau_{t}, t\right)}{\partial X_{t} \partial \tau_{t}}\left(X_{t} \tau_{t} \sigma_{\tau} \rho \sigma_{P, t}\right)\right. \\
& \left.\left.\left.\frac{\partial^{2} J\left(X_{t}, \tau_{t}, t\right)}{\partial \tau_{t}^{2}}\left(\tau_{t}^{2} \sigma_{\tau}^{2} \rho^{2}+\tau_{t}^{2} \sigma_{\tau}^{2}\left(1-\rho^{2}\right)\right)\right]\right] d t \mid F_{t}\right\} .
\end{aligned}
$$

Ahora se divide esta expresión entre $d t$ y se toma el límite cuando $d t \rightarrow 0$ 


$$
\begin{aligned}
& 0=\max _{\theta_{t,}, \theta_{2}, \theta_{3 t}, y \theta_{t t} \in \mathbf{R}, 0 \leq\left. c_{s}\right|_{t, t+t+a]}}\left\{F\left(c_{t}, t\right)+\frac{\partial J\left(X_{t}, \tau_{t}, t\right)}{\partial t}+\frac{\partial J\left(X_{t}, \tau_{t}, t\right)}{\partial X_{t}} X_{t} r_{x} \frac{\partial J\left(X_{t}, \tau_{t}, t\right)}{\partial \tau_{t}} \tau_{t} \bar{\tau}_{t}+\right. \\
& \left.\frac{1}{2}\left[\frac{\partial^{2} J\left(X_{t}, \tau_{t}, t\right)}{\partial X_{t}^{2}}\left(X_{t}^{2} \sigma_{P, t}^{2}+X_{t}^{2} \sigma_{k, t}^{2}\right)+2 \frac{\partial^{2} J\left(X_{t}, \tau_{t}, t\right)}{\partial X_{t} \partial \tau_{t}}\left(X_{t} \tau_{t} \sigma_{\tau} \rho \sigma_{P, t}\right)+\frac{\partial^{2} J\left(X_{t}, \tau_{t}, t\right)}{\partial \tau_{t}^{2}}\left(\tau_{t}^{2} \sigma_{\tau}^{2}\right)\right]\right\} .
\end{aligned}
$$

A la última ecuación se le anexan las condiciones de frontera correspondientes, para obtener la ecuación diferencial parcial de Hamilton-Jacobi-Bellman (HJB):

$$
\left\{\begin{aligned}
\max _{\theta_{t}, \theta_{2 t}, \theta_{3 t}, \theta_{4 t} \mathbf{R}, 0 \leq\left. c_{s}\right|_{[t, t+d t]}}\left\{F\left(c_{t}, t\right)+\frac{\partial J\left(X_{t}, \tau_{t}, t\right)}{\partial t}+\frac{\partial J\left(X_{t}, \tau_{t}, t\right)}{\partial X_{t}} X_{t} r_{x}+\right. \\
\frac{\partial J\left(X_{t}, \tau_{t}, t\right)}{\partial \tau_{t}} \tau_{t} \bar{\tau}_{t}+\frac{1}{2}\left[\frac{\partial^{2} J\left(X_{t}, \tau_{t}, t\right)}{\partial X_{t}^{2}}\left(X_{t}^{2} \sigma_{P, t}^{2}+X_{t}^{2} \sigma_{k, t}^{2}\right)+\right. \\
\left.\left.2 \frac{\partial^{2} J\left(X_{t}, \tau_{t}, t\right)}{\partial X_{t} \partial \tau_{t}}\left(X_{t} \tau_{t} \sigma_{\tau} \rho \sigma_{P, t}\right)+\frac{\partial^{2} J\left(X_{t}, \tau_{t}, t\right)}{\partial \tau_{t}^{2}} \tau_{t}^{2} \sigma_{\tau}^{2}\right]\right\} \\
J(x, \tau, T)=0 \\
J(0, \tau, t)=0 .
\end{aligned}\right.
$$

Se observa que las condiciones de frontera ya incorporan el tiempo de paro.

\section{Función de utilidad}

Se supone ahora que la función de utilidad es de la forma $F\left(c_{t}, t\right)=e^{-\delta t} V\left(c_{t}\right)$, donde $V\left(c_{t}\right)$ es un miembro de la familia de funciones de utilidad HARA (siglas del inglés hyperbolic absolute risk aversion) (Hakansson, 1970; Merton, 1990), en consecuencia,

$$
F\left(c_{t}, t\right)=e^{-\delta t} \frac{c_{t}^{\gamma}}{\gamma}, 0<\gamma<1
$$

Se observa que $V\left(c_{t}\right)$ tiene la propiedad de que

$$
V^{\prime}(0)=\left.\frac{c_{t}^{\gamma}}{c_{t}}\right|_{c_{t}=0}=\infty,
$$


lo que forzará a que el consumo sea positivo a través del horizonte de planeación. Así, al suponer un máximo y hacer las sustituciones de (16) y $F\left(c_{t}, t\right)=e^{-\delta t} V\left(c_{t}\right)$ en la ecuación diferencial parcial de HJB, se tiene que

$$
\begin{aligned}
& 0=e^{-\delta t} \frac{c_{t}^{\gamma}}{\gamma}+\frac{\partial J\left(X_{t}, \tau_{t}, t\right)}{\partial X_{t}} X_{t}\left(\theta_{1 t} r_{m}+\theta_{2 t} r_{b}+\theta_{3 t} r_{k}+\theta_{4 t} r_{V}+\left(1-\sum_{i=1}^{4} \theta_{i t}\right) r_{b}^{*}-\tau_{t}-\left(1+\tau_{c}\right) \frac{c_{t}}{X_{t}}\right)+ \\
& \frac{\partial J\left(X_{t}, \tau_{t}, t\right)}{\partial t}+\frac{\partial J\left(X_{t}, \tau_{t}, t\right)}{\partial \tau_{t}} \tau_{t} \bar{\tau}_{t}+\frac{1}{2}\left[\frac { \partial ^ { 2 } J ( X _ { t } , \tau _ { t } , t ) } { \partial X _ { t } ^ { 2 } } \left(X_{t}^{2}\left(-\sigma_{p} \theta_{1 t}-\sigma_{p} \theta_{2 t}\right)^{2}+\right.\right. \\
& \left.\left.\left.X_{t}^{2}\left(\theta_{3 t} \sigma_{k}+\theta_{4 t} \sigma_{V}\right)^{2}\right)+2 \frac{\partial^{2} J\left(X_{t}, \tau_{t}, t\right)}{\partial X_{t} \partial \tau_{t}}\left(X_{t} \tau_{t} \sigma_{\tau} \rho\left(-\theta_{1 t}-\theta_{2 t}\right) \sigma_{p}\right)+\frac{\partial^{2} J\left(X_{t}, \tau_{t}, t\right)}{\partial \tau_{t}^{2}} \tau_{t}^{2} \sigma_{\tau}^{2}\right]\right\} .
\end{aligned}
$$

Al considerar la restricción cash in advance, se tiene

$$
\begin{aligned}
& 0=e^{-\delta t} \frac{\left(X_{t} \theta_{1 t} \alpha^{-1}\right)^{\gamma}}{\gamma}+\frac{\partial J\left(X_{t}, \tau_{t}, t\right)}{\partial X_{t}} X_{t}\left(\theta_{1 t} r_{m}+\theta_{2 t} r_{b}+\theta_{3 t} r_{k}+\theta_{4 t} r_{V}+\left(1-\sum_{i=1}^{4} \theta_{i t}\right) r_{b}^{*}-\tau_{t}-\left(1+\tau_{c}\right) \frac{\theta_{1 t}}{\alpha}\right) \\
& +\frac{\partial J\left(X_{t}, \tau_{t}, t\right)}{\partial t}+\frac{\partial J\left(X_{t}, \tau_{t}, t\right)}{\partial \tau_{t}} \tau_{t} \bar{\tau}_{t}+\frac{1}{2}\left[\frac { \partial ^ { 2 } J ( X _ { t } , \tau _ { t } , t ) } { \partial X _ { t } ^ { 2 } } \left(X_{t}^{2}\left(\left(-\theta_{1 t}-\theta_{2 t}\right) \sigma_{p}\right)^{2}\right.\right. \\
& \left.\left.\left.+X_{t}^{2}\left(\left(\theta_{3 t} \sigma_{k}+\theta_{4 t} \sigma_{V}\right)\right)^{2}\right)+2 \frac{\partial^{2} J\left(X_{t}, \tau_{t}, t\right)}{\partial X_{t} \partial \tau_{t}}\left(X_{t} \tau_{t} \sigma_{\tau} \rho\left(-\theta_{1 t}-\theta_{2 t}\right) \sigma_{p}\right)+\frac{\partial^{2} J\left(X_{t}, \tau_{t}, t\right)}{\partial \tau_{t}^{2}} \tau_{t}^{2} \sigma_{\tau}^{2}\right]\right\},
\end{aligned}
$$

\section{Condiciones de primer orden}

Ahora, lo que se requiere es optimizar la ecuación de HJB para $c_{t}, \theta_{1 t}, \theta_{2 t}, \theta_{3 t}$ y $\theta_{4 t}$ por lo que se obtienen las condiciones de primer orden:

$$
c_{t}^{\gamma-1}=\frac{\partial J\left(X_{t}, \tau_{t}, t\right)}{\partial X_{t}} e^{\delta t}\left(1+\tau_{c}\right) .
$$

Se nota que la condición de primer orden con respecto de $\theta_{1 t}$, se puede derivar a partir de las ecuaciones (22) y (23), las cuales se describen en las ecuaciones $(24 b)$ y $(24 c)$ respectivamente:

$$
\begin{aligned}
0= & X_{t} e^{-\delta t}\left(\theta_{1 t} \alpha^{-1} X_{t}\right)^{\gamma-1}+\frac{\partial J\left(X_{t}, \tau_{t}, t\right)}{\partial X_{t}}\left(\left(X_{t} r_{m}-X_{t} r_{b}^{*}\right)-X_{t} \frac{\left(1+\tau_{c}\right)}{\alpha}\right)- \\
& \frac{\partial^{2} J\left(X_{t}, \tau_{t}, t\right)}{\partial X_{t} \partial \tau_{t}}\left(X_{t} \tau_{t} \sigma_{\tau} \rho \sigma_{p}\right)-\frac{\partial^{2} J\left(X_{t}, \tau_{t}, t\right)}{\partial X_{t}^{2}} X_{t}^{2} \sigma_{p}\left(-\sigma_{p} \theta_{1 t}-\sigma_{p} \theta_{2 t}\right),
\end{aligned}
$$




$$
\begin{aligned}
0= & \frac{\partial J\left(X_{t}, \tau_{t}, t\right)}{\partial X_{t}}\left(X_{t} r_{m}-X_{t} r_{b}^{*}\right)-\frac{\partial^{2} J\left(X_{t}, \tau_{t}, t\right)}{\partial X_{t} \partial \tau_{t}}\left(X_{t} \tau_{t} \sigma_{\tau} \rho \sigma_{p}\right) \\
& -\frac{\partial^{2} J\left(X_{t}, \tau_{t}, t\right)}{\partial X_{t}^{2}} X_{t}^{2} \sigma_{p}\left(-\sigma_{p} \theta_{1 t}-\sigma_{p} \theta_{2 t}\right), \\
0= & \frac{\partial J\left(X_{t}, \tau_{t}, t\right)}{\partial X_{t}}\left(X_{t} r_{b}-X_{t} r_{b}^{*}\right)-\frac{\partial^{2} J\left(X_{t}, \tau_{t}, t\right)}{\partial X_{t} \partial \tau_{t}}\left(X_{t} \tau_{t} \sigma_{\tau} \rho \sigma_{p}\right) \\
& -\frac{\partial^{2} J\left(X_{t}, \tau_{t}, t\right)}{\partial X_{t}^{2}} X_{t}^{2} \sigma_{p}\left(-\sigma_{p} \theta_{1 t}-\sigma_{p} \theta_{2 t}\right), \\
0= & \frac{\partial J\left(X_{t}, \tau_{t}, t\right)}{\partial X_{t}}\left(X_{t} r_{k}-X_{t} r_{b}^{*}\right)+\frac{\partial^{2} J\left(X_{t}, \tau_{t}, t\right)}{\partial X_{t}^{2}} X_{t}^{2} \sigma_{k}\left(\sigma_{k} \theta_{3 t}+\sigma_{V} \theta_{4 t}\right), \\
0= & \frac{\partial J\left(X_{t}, \tau_{t}, t\right)}{\partial X_{t}}\left(X_{t} r_{V}-X_{t} r_{b}^{*}\right)+\frac{\partial^{2} J\left(X_{t}, \tau_{t}, t\right)}{\partial X_{t}^{2}} X_{t}^{2} \sigma_{V}\left(\sigma_{k} \theta_{3 t}+\sigma_{V} \theta_{4 t}\right) .
\end{aligned}
$$

Ahora para elegir la función $J\left(X_{t}, \tau_{t}, t\right)$ que satisfaga la ecuación diferencial parcial de HJB, se propone como candidato de solución un producto de funciones separables en variables, de tal forma que

$$
J\left(X_{t}, \tau_{t}, t\right)=e^{-\delta t} h\left(\tau_{t}, t\right) \frac{x_{t}^{\gamma}}{\gamma},
$$

junto con $h\left(\tau_{t}, T\right)=0$ debido a las condiciones de frontera de la ecuación HJB. Dado este candidato para $J$, se tiene que

$$
\begin{gathered}
\frac{\partial J\left(X_{t}, \tau_{t}, t\right)}{\partial X_{t}}=e^{-\delta t} h\left(\tau_{t}, t\right) x_{t}^{\gamma-1}, \quad \frac{\partial^{2} J\left(X_{t}, \tau_{t}, t\right)}{\partial X_{t}^{2}}=(\gamma-1) e^{-\delta t} h\left(\tau_{t}, t\right) x_{t}^{\gamma-2}, \\
\frac{\partial J\left(X_{t}, \tau_{t}, t\right)}{\partial \tau_{t}}=e^{-\delta t} \frac{\partial h\left(\tau_{t}, t\right)}{\partial \tau_{t}} \frac{x_{t}^{\gamma}}{\gamma}, \quad \frac{\partial^{2} J\left(X_{t}, \tau_{t}, t\right)}{\partial \tau_{t}^{2}}=e^{-\delta t} \frac{\partial^{2} h\left(\tau_{t}, t\right)}{\partial \tau_{t}^{2}} \frac{x_{t}^{\gamma}}{\gamma}, \\
\frac{\partial J\left(X_{t}, \tau_{t}, t\right)}{\partial t}=e^{-\delta t} x_{t}^{\gamma-1}\left(-\delta h\left(\tau_{t}, t\right)+\frac{\partial h\left(\tau_{t}, t\right)}{\partial t}\right), \quad \frac{\partial^{2} J\left(X_{t}, \tau_{t}, t\right)}{\partial X_{t} \partial \tau_{t}}=e^{-\delta t} \frac{\partial h\left(\tau_{t}, t\right)}{\partial \tau_{t}} x_{t}^{\gamma-1} .
\end{gathered}
$$

Si se sustituyen las derivadas parciales de (26) en las condiciones de primer orden de (24), se obtiene

$$
c_{t}^{\gamma-1}=h\left(\tau_{t}, t\right) x_{t}^{\gamma-1}\left(1+\tau_{c}\right) .
$$


Las demás condiciones de optimidad son:

$$
\begin{aligned}
& 0=h\left(\tau_{t}, t\right)\left(r_{m}-r_{b}^{*}\right)-\frac{\partial h\left(\tau_{t}, t\right)}{\partial \tau_{t}}\left(\tau_{t} \sigma_{\tau} \rho \sigma_{p}\right)+(\gamma-1) h\left(\tau_{t}, t\right) \sigma_{P}^{2}\left(\theta_{1 t}+\theta_{2 t}\right), \\
& 0=h\left(\tau_{t}, t\right)\left(r_{b}-r_{b}^{*}\right)-\frac{\partial h\left(\tau_{t}, t\right)}{\partial \tau_{t}}\left(\tau_{t} \sigma_{\tau} \rho \sigma_{p}\right)+(\gamma-1) h\left(\tau_{t}, t\right) \sigma_{P}^{2}\left(\theta_{1 t}+\theta_{2 t}\right), \\
& 0=X_{t}^{\gamma}\left[h\left(\tau_{t}, t\right)\left(r_{k}-r_{b}^{*}\right)+(\gamma-1) h\left(\tau_{t}, t\right) \sigma_{k}\left(\sigma_{k} \theta_{3 t}+\sigma_{V} \theta_{4 t}\right)\right], \\
& 0=X_{t}^{\gamma}\left[h\left(\tau_{t}, t\right)\left(r_{V}-r_{b}^{*}\right)+(\gamma-1) h\left(\tau_{t}, t\right) \sigma_{V}\left(\sigma_{k} \theta_{3 t}+\sigma_{V} \theta_{4 t}\right)\right] .
\end{aligned}
$$

\section{Decisiones óptimas}

Se puede observar que la trayectoria óptima de consumo $\hat{c}_{t}$ es lineal en la riqueza. En contraste con un marco determinista en donde el individuo puede saber a ciencia cierta cuál será su trayectoria óptima de consumo, en el caso estocástico, desafortunadamente, ésta ya no puede ser determinada porque el consumo se convierte en una variable aleatoria, situación que más acorde con la realidad contingente de la economía en la que el individuo existe, así:

$$
\hat{c}_{t}=h\left(\tau_{t}, t\right)^{1 / x-1} x_{t}\left(1+\tau_{c}\right)^{1 / x-1}
$$

En conclusión, la consideración del riesgo en los diversos factores que constituyen la economía induce cambios tanto cualitativos como cuantitativos importantes en las decisiones de consumo y portafolio. Ahora bien, a partir de (24b) (24c) y (26), se tiene que

$$
\hat{\theta}_{1 t}=\alpha h\left(\tau_{t}, t\right)^{1 / y-1}\left(1+\tau_{c}\right)^{1 / y-1}
$$

En la ecuación (28b) $\hat{\theta}_{1 t}$ es constante, pues sólo depende de los parámetros que determinan las características estocásticas de la economía, en cuyo caso se puede afirmar que la actitud del agente económico hacia el riesgo y, por ende, 
en su toma de decisiones de inversión es independiente de su riqueza. Si ahora se sustituye (28b) en (27c), se tiene que

$$
\begin{aligned}
\hat{\theta}_{2 t}= & -(\gamma-1)^{-1} \sigma_{P}^{-2}\left(r_{b}-r_{b}^{*}\right)-\alpha h\left(\tau_{t}, t\right)^{1 / \gamma-1}\left(1+\tau_{c}\right)^{1 / \gamma-1} \\
& +\frac{\partial h\left(\tau_{t}, t\right)}{\partial \tau_{t}} \tau_{t} \sigma_{\tau} \rho(\gamma-1)^{-1} h\left(\tau_{t}, t\right)^{-1} \sigma_{P}{ }^{-1} .
\end{aligned}
$$

Esta ecuación muestra que $\hat{\theta}_{2 t}$ también es independiente de la riqueza del individuo. Es importante hacer notar que las ecuaciones (27b) y (27c) forman un sistema inconsistente. Obsérvese que al restar (27c) de (27b), se tiene que $0=h\left(\tau_{t}, t\right)\left(r_{m}-r_{b}\right)$, situación que ocurre sólo si $h\left(\tau_{t}, t\right)=0 \mathrm{o} r_{m}-r_{b}=0$, pero $h\left(\tau_{t}, t\right)$ no puede ser cero, porque esa posibilidad lleva a un consumo nulo para optimizar el problema de decisión del agente, lo que es un absurdo; por lo que en el equilibrio sucede $r_{m}-r_{b}=0 \Rightarrow r_{m}=r_{b} \Leftrightarrow 0=i\left(1-\tau_{y}\right)$, que implica que $i=0$, es decir, en esta economía no es óptimo para el gobierno emitir deuda, pero no quiere decir que no sea óptimo para el agente representativo mantener su inversión en bonos.

De las ecuaciones (27d) y (27e) se obtienen las proporciones óptimas para $\theta_{3 t}$ y $\theta_{4 t}$,

$$
\begin{aligned}
& \hat{\theta}_{3 t}=-\frac{\left(r_{k}-r_{b}^{*}\right)+(\gamma-1) \sigma_{k} \sigma_{V} \hat{\theta}_{4 t}}{(\gamma-1) \sigma_{k}^{2}}, \\
& \hat{\theta}_{4 t}=-\frac{\left(r_{V}-r_{b}^{*}\right)+(\gamma-1) \sigma_{k} \sigma_{V} \hat{\theta}_{3 t}}{(\gamma-1) \sigma_{V}^{2}} .
\end{aligned}
$$

Nótese que estas dos ecuaciones forman un sistema redundante:

$$
\begin{aligned}
& \hat{\theta}_{3 t}+\frac{\sigma_{V} \theta_{4 t}}{\sigma_{k}}=-\frac{\left(r_{k}-r_{b}^{*}\right)}{(\gamma-1) \sigma_{k}^{2}}=\lambda_{k} \\
& \hat{\theta}_{4 t}+\frac{\sigma_{k} \theta_{3 t}}{\sigma_{v}}=-\frac{\left(r_{V}-r_{b}^{*}\right)}{(\gamma-1) \sigma_{V}^{2}}=\lambda_{V}
\end{aligned}
$$

al hacer $\zeta=\frac{\sigma_{V}}{\sigma_{k}}$, se tiene

$$
\begin{aligned}
& \hat{\theta}_{3 t}+\zeta \hat{\theta}_{4 t}=\lambda_{k}, \\
& \frac{\hat{\theta}_{3 t}}{\zeta}+\hat{\theta}_{4 t}=\lambda_{V},
\end{aligned}
$$

lo cual implica que 
90 ECONOMÍA: TEORÍA Y PRÁCTICA • Nueva Época, número 41, julio-diciembre 2014

$$
\underbrace{\left(\begin{array}{cc}
1 & \zeta \\
\zeta^{-1} & 1
\end{array}\right)}_{A}\left(\begin{array}{c}
\hat{\theta}_{3 t} \\
\hat{\theta}_{4 t}
\end{array}\right)=\left(\begin{array}{c}
\lambda_{k} \\
\lambda_{V}
\end{array}\right) .
$$

Sin embargo, de (31) se tiene que $\operatorname{det}(A)=0$, por lo que el sistema no tiene una única, sino una infinidad de soluciones. Esto quiere decir que los premios al riesgo de mercado para la acción y para la opción son combinación lineal uno del otro, lo cual es lógico porque la opción hereda propiedades del proceso de precios del subyacente.

\section{Premio Al RIESgo de Mercado de Una opción AMERICANa DE VENTA}

A partir del sistema de ecuaciones (31), se tiene que

$$
\lambda_{k}=\zeta \lambda_{V}
$$

es decir, los premios al riesgo del mercado del subyacente y de la opción coinciden, lo que se corrobora de (29), con lo cual se obtiene:

$$
\left(r_{k}-r_{b}^{*}\right) \frac{\sigma_{v}}{\sigma_{k}}=\left(r_{V}-r_{b}^{*}\right) .
$$

Después de hacer las sustituciones de $r_{V}$ y $\sigma_{V}$, que se definen en (7a), se obtiene,

$$
\frac{\partial V_{t}}{\partial t}+\frac{1}{2} \frac{\partial^{2} V_{t}}{\partial S_{t}^{2}} \sigma_{k}^{2} S_{t}^{2}+\frac{\partial V_{t}}{\partial S_{t}} r_{b}^{*} S_{t}-V_{t} r_{b}^{*}=0
$$

la cual es la ecuación diferencial parcial de Black-Scholes (1973) y Merton (1973), con la que se valuará la opción americana y a la que para tal efecto se deben de imponer las condiciones de frontera correspondientes a la opción americana de venta $\mathrm{y}$ al límite estocástico de tiempo de paro $\varphi=\min \left[\inf \left\{t>0 \mid X_{t}=0\right\}, T\right]$, esto es,

$$
\begin{aligned}
& \frac{\partial V_{t}}{\partial t}+\frac{1}{2} \frac{\partial^{2} V_{t}}{\partial S_{t}^{2}} \sigma_{k}^{2} S_{t}^{2}+\frac{\partial V_{t}}{\partial S_{t}} r_{b}^{*} S_{t}-V_{t} r_{b}^{*}=0 \\
& V\left(S_{t}, t\right) \geq \max \left(K-S_{t}, 0\right) \\
& t \leq \bar{\varphi},
\end{aligned}
$$


en donde $K$ denota el precio de ejercicio de la opción americana en el tiempo de ejercicio $\bar{\varphi}=\min \{\varphi, \hat{\varphi}\}$, donde $\hat{\varphi}$ es un tiempo de paro tal que se alcanza el valor $\max \left(K-S_{t}, 0\right)$. Obsérvese que la tasa de interés libre de riesgo es $r_{b}^{*}$, dado que la economía doméstica es precio aceptante y toma la tasa de interés internacional como dada, ya que en la economía doméstica la tasa real $r_{b}$ se calcula en términos del diferencial de la tasa de interés nominal y la tasa de inflación, y en esta última hay factores de riesgo que la afectan, como el nivel general de precios.

\section{Caracterización del PRecio de Una OPCión americana DE VENTA}

La prima de la opción americana es más cara que la de una opción europea, ya que aquélla tiene la flexibilidad de ser ejercida en cada instante desde la emisión del contrato de opción y hasta el tiempo de madurez de la misma, lo que dota al poseedor del contrato del derecho de obtener ganancias mayores que cuando se trata de una opción europea, toda vez que en el ejercicio anticipado existe la posibilidad de obtener mejores ganancias. Esto hace del instrumento americano un activo de inversión atractivo para quien posee el contrato ${ }^{11}$ (Wilmott, Howison y Dewynne 1999).

Es posible observar que en el tipo de problema de control óptimo que se está considerando, el tiempo de madurez es estocástico; lo anterior exige que el derivado obtenido pueda ser ejercido en cualquier momento. Ésta resulta ser, precisamente una característica de la opción americana. En el problema de valuación de la opción americana, no sólo se debe de determinar su valor en cada instante, sino también hay que especificar si se ejerce o no la opción para cada valor de $k_{t}$. Usualmente, esto se lleva a cabo estableciendo un valor crítico de $k^{*}$ para cada $t$.

Sin pérdida de generalidad, se supone que dicho valor es único. Por lo que si $k_{t} \leq k^{*}$, se está en la región de ejercicio y es óptimo ejercer la opción, y si $k_{t}>k^{*}$, no se está en la región de ejercicio y lo óptimo es mantener la opción Venegas-Martínez (2008). De esta manera, si la opción es ejercida cuando $k_{t}>k^{*}$, entonces el precio de la opción americana de venta, $V=V_{t}\left(k_{t}, t\right)$, satisface la ecuación diferencial parcial:

\footnotetext{
${ }^{11}$ Aunque no así para su emisor.
} 
92 ECONOMÍA: TEORÍA Y PRÁCTICA • Nueva Época, número 41, julio-diciembre 2014

$$
\frac{\partial V_{t}}{\partial t}+\frac{1}{2} \frac{\partial^{2} V_{t}}{\partial k_{t}^{2}} \sigma_{k}^{2} k_{t}^{2}+\frac{\partial V_{t}}{\partial k_{t}} r_{b}^{*} k_{t}-V_{t} r_{b}^{*}=0
$$

Dado que una opción americana es más cara que una europea, denotemos por $\varepsilon\left(k_{t}, t\right)$ el premio por el ejercicio anticipado de la opción. Entonces,

$$
V_{t}\left(k_{t}, t\right)=p_{B S M}\left(k_{t}, t\right)+\varepsilon\left(k_{t}, t\right)
$$

o

$$
\varepsilon\left(k_{t}, t\right)=V_{t}\left(k_{t}, t\right)-p_{B S M}\left(k_{t}, t\right)
$$

donde $p_{B S M}\left(k_{t}, t\right)$ es el precio de una opción europea de venta que satisface la ecuación diferencial parcial lineal de segundo orden de Black-Scholes-Merton, $B S M$. Toda vez que $V_{t}\left(k_{t}, t\right)$ también satisface una ecuación análoga a (34) (con sus correspondientes condiciones de frontera), se tiene que

$$
\frac{\partial \varepsilon}{\partial t}+\frac{1}{2} \frac{\partial^{2} \varepsilon}{\partial k_{t}^{2}} \sigma_{k}^{2} k_{t}^{2}+\frac{\partial \varepsilon}{\partial k_{t}} r_{b}^{*} k_{t}-\varepsilon r_{b}^{*}=0 .
$$

Si se define el cambio de variable

$$
u=\bar{\tau}-t
$$

junto con los parámetros

$$
M=2 r_{b}^{*} \sigma_{k}^{-2} \quad \text { y } \quad G=2 r_{b}^{*} \sigma_{k}^{-2}-1,
$$

al multiplicar la ecuación (34) por el parámetro $M$, e incluir el cambio de variable, se puede expresar (37) como

$$
\begin{aligned}
0 & =M \frac{\partial \varepsilon}{\partial u} \frac{\partial u}{\partial t}+2 r_{b}^{*} \sigma_{k}^{-2} \frac{1}{2} \frac{\partial^{2} \varepsilon}{\partial k_{t}^{2}} \sigma_{k}^{2} k_{t}^{2}+2 r_{b}^{*} \sigma_{k}^{-2} \frac{\partial \varepsilon}{\partial k_{t}} r_{b}^{*} k_{t}-M \varepsilon r_{b}^{*} \\
& =-M \frac{\partial \varepsilon}{\partial u}+r_{b}^{*} \frac{\partial^{2} \varepsilon}{\partial k_{t}^{2}} k_{t}^{2}+2 r_{b}^{* 2} \sigma_{k}^{-2} \frac{\partial \varepsilon}{\partial k_{t}} k_{t}-M \varepsilon r_{b}^{*}
\end{aligned}
$$

Si ahora se divide entre $r_{b}^{*}$ y se hacen las sustituciones correspondientes, se tiene

$$
0=-\frac{M}{r_{b}^{*}} \frac{\partial \varepsilon}{\partial u}+\frac{\partial^{2} \varepsilon}{\partial k_{t}^{2}} k_{t}^{2}+(G+1) \frac{\partial \varepsilon}{\partial k_{t}} k_{t}-M \varepsilon
$$


Si se supone una solución de la forma $\varepsilon\left(k_{t}, t\right)=g(u) f\left(k_{t}, g(u)\right)$ con $f\left(k_{t}, 0\right)=0$, entonces

$$
\frac{\partial \varepsilon}{\partial k_{t}}=g \frac{\partial f}{\partial k_{t}}, \quad \frac{\partial^{2} \varepsilon}{\partial k_{t}^{2}}=g \frac{\partial^{2} f}{\partial k_{t}^{2}} \quad \text { y } \quad \frac{\partial \varepsilon}{\partial u}=f \frac{d g}{d u}+\frac{\partial f}{\partial g} g \frac{d g}{d u}=\frac{d g}{d u}\left(f+\frac{\partial f}{\partial g} g\right) \text {. }
$$

Al sustituir (37) en (34) y factorizar $g$, se sigue que

$$
0=-\frac{M}{r_{b}^{*}} \frac{d g}{d u}\left(\frac{f}{g}+\frac{\partial f}{\partial g}\right)+\frac{\partial^{2} f}{\partial k_{t}^{2}} k_{t}^{2}+(G+1) \frac{\partial f}{\partial S_{t}} k_{t}-M f
$$

o

$$
0=\frac{\partial^{2} f}{\partial k_{t}^{2}} k_{t}^{2}+(G+1) \frac{\partial f}{\partial k_{t}} k_{t}-M f\left(1+\frac{1}{r_{b}^{*} g} \frac{d g}{d u}\left(1+\frac{g}{f} \frac{\partial f}{\partial g}\right)\right)
$$

Si se elige $g(u)=1-e^{-r u}$, entonces

$$
-M f\left(1+\frac{1}{r_{b}^{*} g} \frac{d g}{d u}\left(1+\frac{g}{f} \frac{\partial f}{\partial g}\right)\right)=-M f\left(1+\frac{1-g(u)}{g}\left(1+\frac{g}{f} \frac{\partial f}{\partial g}\right)\right) .
$$

En consecuencia, (38) se transforma en

$$
0=\frac{\partial^{2} f}{\partial k_{t}^{2}} k_{t}^{2}+(G+1) \frac{\partial f}{\partial k_{t}} k_{t}-(1-g) M \frac{\partial f}{\partial g}-\frac{M}{g} f .
$$

Y se tiene entonces que $V_{t}\left(k_{t}, t\right)=\varepsilon\left(k_{t}, t\right)+p_{B S M}\left(k_{t}, t\right), k_{t}<k^{*}$, donde el primer sumando es $\varepsilon\left(k_{t}, t\right)=g(u) f\left(k_{t}, g(u)\right)$ con $g(u)=1-e^{-r u}$ y $f\left(k_{t}, g(u)\right)$ satisface (41), y el segundo sumando es la solución tradicional del modelo de Black-Scholes-Merton. De esta manera, si el activo subyacente es conducido por el movimiento geométrico browniano en un mundo neutral al riesgo, entonces el precio $V_{t}\left(k_{t}, t\right)$ se calcula en términos del premio al riesgo, el cual se calcula a través de una ecuación diferencial parcial lineal. Esta propuesta es una alternativa a los modelos más populares en la literatura financiera para valuar opciones americanas con fórmulas aproximadas, como son los de Whaley (1981) y Barone-Adesi y Whaley (1987). 
94 ECONOMÍA: TEORÍA Y PRÁCTICA • Nueva Época, número 41, julio-diciembre 2014

\section{APROXIMACIÓN DEL PRECIO DE UNA OPCIÓN AMERICANA DE VENTA Y ANÁLISIS DE SENSIBILIDAD RESPECTO DE SUS PARÁMETROS}

\section{Aproximación del precio de una opción americana de venta}

En esta sección, primeramente, se obtiene una aproximación del precio de la opción americana y después se realiza un análisis de sensibilidad de éste con respecto a sus parámetros. Un primer resultado interesante es que no depende de parámetros estructurales del modelo, como el impuesto sobre la riqueza, $\tau_{t}$; la tasa media esperada del impuesto sobre la riqueza, $\bar{t}_{t}$; la volatilidad asociada, $\sigma_{\tau}$, al impuesto sobre la riqueza, ni del parámetro $\alpha$, que representa el tiempo que está disponible el dinero para financiar el consumo.

Asimismo, se observa que cuando $u \rightarrow 0$, donde $u=\bar{\tau}-t$, entonces $(1-g) \rightarrow 0$. Así, cuando el tiempo de vencimiento crece, el último término de la ecuación (41) es insignificante, por lo que entonces se tiene que

$$
\lim _{u \rightarrow 0} \frac{\partial \varepsilon}{\partial u}=\lim _{u \rightarrow 0} f \frac{\partial g}{\partial u}=\lim _{u \rightarrow 0} f\left(k_{t}, 1-e^{-r u}\right)\left(r e^{-r u}\right)=r f\left(k_{t}, 0\right)=0,
$$

lo que permite eliminar el penúltimo término de la ecuación (41), de lo que se obtiene

$$
0=\frac{\partial^{2} f}{\partial k_{t}^{2}} k_{t}^{2}+(G+1) \frac{\partial f}{\partial k_{t}} k_{t}-\frac{M}{g} f
$$

La anterior es una ecuación diferencial ordinaria de segundo orden en $f$, y se supone una solución de la forma $f\left(k_{t}, g(u)\right)=a k_{t}^{q(g)}$. En consecuencia,

$$
\frac{\partial f}{\partial k_{t}} a q(g) k_{t}^{q(g)-1} \quad \text { y } \quad \frac{\partial^{2} f}{\partial k_{t}^{2}} a q(g)(q(g)-1) k_{t}^{q(g)-2} .
$$

Al sustituir $f\left(k_{t}, g(u)\right)=a k_{t}^{q(g)}$ y (43) en (42), se tiene que

$$
a k_{t}^{q(g)}\left[q^{2}(g)+G q(g)-M / g\right]=0 .
$$

A partir del polinomio característico de (44) se obtiene 


$$
q_{1}(g)=-G-\sqrt{G^{2}-4(M / G)} \quad \text { y } \quad q_{2}(g)=-G+\sqrt{G^{2}-4(M / G)}
$$

de donde $q_{1}<0$ y $q_{2}>0$, siempre que $M / \mathrm{g}>0$. Así, la solución general de la ecuación diferencial en (42) está dada por

$$
f\left(k_{t}, g(u)\right)=c_{1} k_{t}^{q_{1}(g)}+c_{2} k_{t}^{q_{2}(g)}
$$

con $c_{1} \mathrm{y} c_{2}$, constantes por determinar. A partir de (46) se observa, por una parte, que dado $q_{2}>0$, si $k_{t}$ crece muy rápido, sería entonces factible rebasar el precio crítico $k^{*}$, lo que conduce fuera de la región de ejercicio y, por tanto, no es óptimo el ejercicio anticipado y la prima de la opción americana resulta ser igual a la de la europea. Por otra parte, si $k_{t}$ crece rápidamente, entonces $\varepsilon$ también crece de la misma manera, con lo que es óptimo ejercer anticipadamente (de inmediato). Estos dos resultados generan una contradicción, por lo que se debe escoger $c_{2}=0$. Así, el premio por el ejercicio anticipado está definido únicamente por el primer término de $f\left(k_{t}, g(u)\right)$.

Se reformula, entonces, el precio $V_{t}\left(k_{t}, t\right)$ de la opción americana de venta dado en (35) como

$$
V_{t}\left(k_{t}, t\right)=p_{B S M}\left(k_{t}, t\right)+c_{1} g(u) k_{t}^{q_{1}(g)} .
$$

Se observa que a medida que $k_{t}$ decrece, $V_{t}\left(k_{t}, t\right), p_{B S M}\left(k_{t}, t\right)$ y $c_{1} g(u) k_{t}^{q_{t}(g)}$ decrecen. En particular, el crecimiento de $c_{1} g(u) k_{t}^{q_{1}(g)}$ depende del signo de $c_{1}$, pero para que tenga sentido el ejercicio anticipado se debe tener $c_{1}>0$, ya que de lo contrario, el precio de la prima de la opción americana sería más barato que el de la opción europea y convendría al poseedor del contrato no ejercer anticipadamente. Sean $k^{*}$ y $c_{1}>0$ tales que

$$
\kappa-k^{*}=p_{B S M}\left(k^{*}, u\right)+c_{1} g(u)\left(k^{*}\right)^{q_{1}(g)}
$$

con $\kappa$ precio de ejercicio y $k^{*}$ precio crítico por el ejercicio anticipado. $\mathrm{Si}$ $k_{t} \leq k^{*}$, entonces

$$
\kappa-k_{t} \geq p_{B S M}\left(k^{*}, u\right)+c_{1} g(u)\left(k^{*}\right)^{q_{1}(g)},
$$


y el precio de la opción americana es $V_{t}\left(k_{t}, t\right)=\kappa-k_{t}$ pero si $k_{t}>k$, entonces se satisface (47). Al derivar (48) de ambos lados del igual respecto de $k^{*}$ se obtiene

$$
-1=-e^{-r u} \Phi\left(-d_{1}\left(k^{*}\right)\right)+c_{1} g q_{1}(u)\left(k^{*}\right)^{\left(q_{1}-1\right)(g)}
$$

donde

$$
d_{1}\left(k^{*}\right)=\frac{\ln \left(\frac{k^{*}}{\kappa}\right)+\left(r_{b}^{*}+\frac{1}{2} \sigma_{k}^{2}\right) u}{\sigma_{k} \sqrt{u}}
$$

y $\Phi(\cdot)$ es la función de distribución acumulada de una variable aleatoria normal estándar. A partir de (48) y (50), se tiene que

$$
\left\{\begin{array}{l}
\kappa-k^{*}=p_{B S M}\left(k^{*} u\right)+\frac{\left\{-1+e^{-r_{b}^{*} u} \Phi\left(-d_{1}\left(k^{*}\right)\right)\right\} k^{*}}{q_{1}} \\
c_{1}=\frac{-1+e^{-r_{b}^{*} u} \Phi\left(-d_{1}\left(k^{*}\right)\right)}{q_{1} g(u)\left(k^{*}\right)^{\left(q_{1}-1\right)(g)}}
\end{array}\right.
$$

Una vez que $k^{*}$ es determinada, se calcula el valor de $c_{1}$. Por lo tanto,

$$
V_{t}\left(k_{t}, t\right)=\left\{\begin{array}{lll}
p_{B S M}\left(k_{t}, u\right)+\left(\left\{-1+e^{-r_{b}^{*} u} \Phi\left(-d_{1}\left(k^{*}\right)\right)\right\} k^{*} / q_{1}\right)\left(\frac{k_{t}}{k^{*}}\right)^{q_{1}}, & \text { si } k_{t} \leq k^{*} \\
\kappa-k_{t}, & \text { si } k_{t}>k^{*}
\end{array},\right.
$$

donde $0<\Phi\left(-d_{1}\left(k^{*}\right)\right)<1$.

\section{Análisis de sensibilidad del precio de una opción americana de venta} respecto de sus parámetros

Se reescribe la ecuación (52) de la siguiente manera,

$$
V_{t}\left(k_{t}, t\right)= \begin{cases}\left\{\kappa e^{-r_{b}^{*} u} \Phi\left(-d_{2}\left(k_{t}\right)\right)-k_{t} \Phi\left(-d_{1}\left(k_{t}\right)\right)\right\} & \\ +\left(\left\{-1+e^{-r_{b}^{*} u} \Phi\left(-d_{1}\left(k^{*}\right)\right)\right\} k^{*} / q_{1}\right)\left(\frac{k_{t}}{k^{*}}\right)^{q_{1}}, & \text { si } k \leftrightharpoons k^{*}, \\ \kappa-k_{t}, & \text { si } k_{t}>k^{*}\end{cases}
$$


donde

$$
d_{1}\left(k_{t}\right)=\frac{\ln \left(\frac{k_{t}}{\kappa}\right)+\left(r_{b}^{*}+\frac{1}{2} \sigma_{k}^{2}\right) u}{\sigma_{k} \sqrt{u}} \quad \text { y } \quad d_{2}\left(k_{t}\right)=d_{1}-\sigma_{k} \sqrt{u} .
$$

a) Razón de cambio entre el precio de la opción americana de venta y el precio del subyacente

A continuación, se denota como $\Delta_{V} \equiv \frac{\partial V}{\partial k_{t}}$ a la razón de cambio entre el precio de la opción americana de venta y el precio de la acción, la cual se calcula como

$$
\begin{aligned}
\frac{\partial V_{t}}{\partial k_{t}}= & -\Phi\left(-d_{1}\left(k_{t}\right)\right)-k_{t} \Phi^{\prime}\left(-d_{1}\left(k_{t}\right)\right)\left(-\frac{\partial d_{1}}{\partial k_{t}}\right)+\kappa e^{-r_{b}^{*} u} \Phi^{\prime}\left(-d_{2}\left(k_{t}\right)\right)\left(-\frac{\partial d_{2}}{\partial k_{t}}\right) \\
& +\left(\frac{k_{t}}{k^{*}}\right)^{q_{1}-1}\left[e^{-r_{b}^{*} u} \Phi\left(-d_{1}\left(k^{*}\right)\right)-1\right],
\end{aligned}
$$

de donde

$$
\begin{aligned}
\Delta_{V} \equiv \frac{\partial V_{t}}{\partial k_{t}}= & \frac{1}{k_{t} \sigma_{k} \sqrt{u}}\left[k_{t} \Phi^{\prime}\left(-d_{1}\left(k_{t}\right)\right)-\kappa e^{-r_{b}^{*} u} \Phi^{\prime}\left(-d_{2}\left(k_{t}\right)\right)\right] \\
& +\left(\frac{k_{t}}{k^{*}}\right)^{q_{1}-1}\left[e^{-r_{b}^{*} u} \Phi\left(-d_{1}\left(k^{*}\right)\right)-1\right]-\Phi\left(-d_{1}\left(k_{t}\right)\right)
\end{aligned}
$$

si se consideran las propiedades siguientes:
a) $\Phi(d)=1-\Phi(-d)$
b) $\Phi^{\prime}(d)=\Phi^{\prime}(-d)$
c) $k_{t} \Phi^{\prime}\left(d_{1}\right)-\kappa e^{-r_{b}^{*} u} \Phi^{\prime}\left(d_{2}\right)=0$,

donde

$$
\Phi^{\prime}(d)=\frac{1}{\sqrt{2 \pi}} e^{-\frac{1}{2} d^{2}}
$$

Obsérvese que la propiedad c) corresponde al lema fundamental de las griegas del modelo de Black-Scholes. Así, después de aplicar la propiedad c) se obtiene 


$$
\Delta_{V} \equiv \begin{cases}\frac{\partial V_{t}}{\partial k_{t}}=\left(\frac{k_{t}}{k^{*}}\right)^{q_{1}-1}\left[e^{-r_{b}^{*} u} \Phi\left(-d_{1}\left(k^{*}\right)\right)-1\right]-1+\Phi\left(d_{1}\left(k_{t}\right)\right) & \text { si } k_{t} \leq k^{*}, \\ \frac{\partial V_{t}}{\partial k_{t}}=-1 & \text { si } k_{t}>k^{*}\end{cases}
$$

A partir de (56) se deduce que $\Delta_{V}<0$, es decir, existe una relación inversa entre el precio de la opción americana de venta y el precio del activo subyacente. Para examinar que en la región de ejercicio $\Delta_{V}<0$, observe que $\left(\frac{k_{t}}{k^{*}}\right)^{q_{1}-1}>0,0<e^{-r_{b}^{*} u}<1$, ya que $r_{b}^{*}>0$. Es de notar que $0<\Phi\left(-d_{1}\left(k^{*}\right)\right)<1$, lo cual se estableció así a partir de la aproximación al precio de la opción americana de venta y $0<\Phi\left(d_{1}\left(k_{t}\right)\right)<1$; esto último debido a que $\Phi$ es una función de distribución.

\section{b) Variación del precio de la opción americana de venta con respecto a la tasa de interés}

La variación del precio de la opción americana de venta $V_{t}\left(k_{t}, t\right)$ con respecto de la tasa de interés $r_{b}^{*}$ esta dada por la siguiente expresión:

$$
\begin{aligned}
\frac{\partial V_{t}}{\partial r_{b}^{*}}= & -\kappa u e^{-r_{b}^{*} u} \Phi\left(-d_{2}\left(k_{t}\right)\right)+\kappa e^{-r_{b}^{*} u} \Phi^{\prime}\left(-d_{2}\left(k_{t}\right)\right)\left(-\frac{\partial d_{2}}{\partial r_{b}^{*}}\right)-k_{t} \Phi^{\prime}\left(-d_{1}\left(k_{t}\right)\right)\left(-\frac{\partial d_{1}}{\partial r_{b}^{*}}\right) \\
& +\left(\frac{k_{t}}{k^{*}}\right)^{q_{1}}\left(\frac{k^{*}}{q_{1}}\right)\left[e^{-r_{b}^{*} u} \Phi^{\prime}\left(-d_{1}\left(k^{*}\right)\right)\left(-\frac{\partial d_{1}}{\partial r_{b}^{*}}\right)-u e^{-r_{b}^{*} u} \Phi\left(-d_{1}\left(k^{*}\right)\right)\right]
\end{aligned}
$$

en donde, después de aplicar las propiedades en (55), se obtiene

$$
\begin{aligned}
\frac{\partial V_{t}}{\partial r_{b}^{*}}= & \left(\frac{k_{t}}{k^{*}}\right)^{q_{1}}\left(\frac{k^{*}}{q_{1}}\right)(-u)\left[e^{-r_{b}^{*} u} \Phi^{\prime}\left(-d_{1}\left(k^{*}\right)\right)\left(\frac{1}{\sigma_{k} \sqrt{u}}\right)-e^{-r_{b}^{*} u} \Phi\left(-d_{1}\left(k^{*}\right)\right)\right] \\
& -\kappa u e^{-r_{b}^{*} u} \Phi\left(-d_{2}\left(k_{t}\right)\right)
\end{aligned}
$$

La ecuación (57) conduce a $\frac{\partial V_{t}}{\partial r_{b}^{*}}<0$ siempre que $k_{t} \leq k^{*}$, es decir, la relación del precio de la opción americana de venta es inversa respecto de la tasa 
de interés. Obsérvese que $\left(\frac{k_{t}}{k^{*}}\right)^{q_{1}}\left(\frac{k^{*}}{q_{1}}\right)(-u)>0$, porque $q_{1}<0, \Phi^{\prime}\left(-d_{1}\left(k^{*}\right)\right)$ $=-\Phi^{\prime}\left(d_{1}\left(k^{*}\right)\right)$, y $0<\Phi\left(-d_{1}\left(k^{*}\right)\right)<1$. Por lo tanto, $\left[e^{-r_{b}^{*} u} \Phi^{\prime}\left(-d_{1}\left(k^{*}\right)\right)\left(\frac{1}{\sigma_{k} \sqrt{u}}\right)\right.$ $\left.-e^{-r_{b}^{*} u} \Phi\left(-d_{1}\left(k^{*}\right)\right)\right]<0$, y a partir de la propiedad a) de (55), se obtiene que $0<\Phi\left(-d_{2}\left(k_{t}\right)\right)<1$.

\section{c) Variación del precio de la opción americana de venta con respecto al precio de ejercicio}

La variación del precio de la opción americana de venta $V_{t}\left(k_{t}, t\right)$ con respecto del precio de ejercicio $\kappa$ se calcula con base en $\frac{\partial d_{2}}{\partial \kappa}=\frac{\partial d_{1}}{\partial \kappa}=\frac{1}{\sigma_{k} \sqrt{u}}\left(-\frac{1}{k_{t} \kappa}\right)$ y la aplicación del lema fundamental de las griegas de Black-Scholes, obteniendo la siguiente expresión:

$$
\frac{\partial V_{t}}{\partial \kappa}= \begin{cases}e^{-r_{b}^{*} u} \Phi\left(-d_{2}\left(k_{t}\right)\right)+\left(\frac{k_{t}}{k^{*}}\right)^{q_{1}}\left(\frac{k^{*}}{q_{1}}\right)\left(\frac{1}{\sigma_{k} \sqrt{u}}\right)\left(-e^{-r_{b}^{*} u} \Phi^{\prime}\left(d_{1}\left(k^{*}\right)\right)\right) & \text { si } k_{t} \leq k^{*}, \\ 1, & \text { si } k_{t}>k^{*} .\end{cases}
$$

Se observa que en todo momento $\frac{\partial V_{t}}{\partial \kappa}>0$, porque a partir de la ecuación (58) se tiene que el primer sumando es positivo, dado que $\Phi(-d)=1-\Phi(d)$, pero $0<\Phi(d)<1$, por ser $\Phi(d)$ una función de distribución. Por otra parte, el segundo sumando también es positivo ya que $\Phi^{\prime}\left(d_{1}\left(k^{*}\right)\right)>0$, lo cual se justifica mediante la ecuación (55), ya que $\left(\frac{1}{\sigma_{k} \sqrt{u}}\right)>0$, dado que $\sigma_{k}>0$. En virtud de que $q_{1}<0$, se tiene producto de signos negativos, lo que hace que la expresión resultante sea positiva. Los demás factores en (58) son positivos, de modo que se confirma que la relación del precio de la opción europea de venta con respecto del precio de ejercicio es positiva.

\section{VERIFICACIÓN DE LA SOLUCIÓN CON PROGRAMACIÓN DINÁMICA}

Para hacer uso del teorema de verificación hay que encontrar la función $h\left(\tau_{t}, t\right)$ definida en $J\left(X_{t}, \tau_{t}, t\right)$ que resuelve la ecuación de HJB. Para esto, se observa que la regla óptima de consumo $\hat{c}_{t}$ es lineal en la riqueza y $\hat{\theta}_{1 t}, \hat{\theta}_{2 t}, \hat{\theta}_{3 t}$ y $\hat{\theta}_{4 t}$ son cons- 
tantes. Además, se supone una solución de esquina para las proporciones óptimas asignadas a la tenencia del activo riesgoso y a su opción americana, de tal forma que $\hat{\theta}_{3 t}=0$ y $\hat{\theta}_{4 t}=1$. La interpretación correspondiente de esta solución de esquina es que si el agente mantiene la opción americana, entonces cuánto estaría él dispuesto a pagar por dicha opción, al mismo tiempo que maximiza su satisfacción por el consumo. Para tal efecto, se sustituyen en la ecuación (22) los valores obtenidos en las ecuaciones (3) (4) (7a) (16) (26) (27a) (28a) (28b) (28c) y (28d), y las derivadas parciales en $\mu_{V}$ y $\sigma_{V}$ se evalúan en el dinero, es decir, $k_{t}=K$, las cuales se denotan mediante

$$
\left.\mu_{V}\right|_{k_{t}=K}=\bar{\mu}_{V} \quad \text { y }\left.\quad \sigma_{V}\right|_{k_{t}=K}=\bar{\sigma}_{V}
$$

de donde se obtiene

$$
\begin{aligned}
& 0=X^{\gamma}\left\{\frac{h\left(\tau_{t}, t\right)^{\gamma / \gamma-1}\left(1+\tau_{c}\right)^{y / \gamma-1}}{\gamma}+h\left[\left(\hat{\theta}_{1 t}+\hat{\theta}_{2 t}\right)\left(-\pi+\sigma_{P}^{2}\right)+\hat{\theta}_{2 t} i\left(1+\tau_{y}\right)\right.\right. \\
& \left.+r_{b}^{*}\left(1-\hat{\theta}_{1 t}+\hat{\theta}_{2 t}\right)+\frac{r_{b}^{*} r_{V}-r_{b}^{2 *}}{(\gamma-1) \sigma_{V}^{2}}-\tau_{t}-h\left(\tau_{t}, t\right)^{1 / \gamma-1}\left(1+\tau_{c}\right)^{-\gamma / \gamma-1}\right]+\frac{\partial h\left(\tau_{t}, t\right)}{\partial \tau_{t}} \tau_{t} \bar{\tau}_{t} \\
& +\frac{1}{\gamma}\left(\frac{\partial h\left(\tau_{t}, t\right)}{\partial t}-\delta h\left(\tau_{t}, t\right)\right)+\frac{1}{2}\left\{( \gamma - 1 ) h ( \tau _ { t } , t ) \left[-\sigma_{P}^{2}\left(\hat{\theta}_{1 t}^{2}+2 \hat{\theta}_{1 t} \hat{\theta}_{2 t}+\hat{\theta}_{2 t}^{2}\right)+\right.\right. \\
& \left.\left.+\frac{r_{V}^{2}-2 r_{b}^{*} r_{V}+r_{b}^{2 *}}{(\gamma-1)^{2} \sigma_{V}^{2}}\right]-2 \frac{\partial h\left(\tau_{t}, t\right)}{\partial \tau_{t}} \tau_{t} \sigma_{\tau} \rho \sigma_{P}\left(\hat{\theta}_{1 t}+\hat{\theta}_{2 t}\right)+\frac{\partial^{2} h\left(\tau_{t}, t\right)}{\partial \tau_{t}^{2}} \tau_{t}^{2} \sigma_{\tau}^{2} \frac{1}{\gamma}\right\} .
\end{aligned}
$$

$\mathrm{Al}$ resolver (59) y obtener la forma funcional $h\left(\tau_{t}, t\right)$, se establecen de manera analítica las trayectorias de $\hat{c}_{t}, \hat{\theta}_{1 t}$ y $\hat{\theta}_{2 t}$.

\section{CONCLUSiones}

El objetivo central de esta investigación fue el de desarrollar un modelo de equilibrio general dinámico estocástico de una economía pequeña y abierta, para determinar en términos de la racionalidad económica cuánto estaría dispuesto a pagar un individuo por una opción americana de venta cuando se enfrenta a la decisión de distribuir su riqueza entre consumo e inversión en un portafolio de 
activos si el horizonte temporal del que dispone es finito con fecha final estocástica; todo lo anterior en un ambiente política fiscal incierta.

Es pertinente mencionar que aun cuando en la literatura existe un número importante de trabajos que aproximan y valúan los precios de primas de opciones americanas mediante diversas metodologías, hasta el momento no se había desarrollado la valuación de estos instrumentos derivados mediante control óptimo estocástico en términos de racionalidad económica.

Se observa en el modelo propuesto que al suponer como parte de los activos de inversión del agente económico una opción americana de venta y caracterizar analíticamente los supuestos relevantes del modelo mediante procesos de difusión observables conducidos por el movimiento geométrico browniano en un mundo neutral al riesgo, se logra caracterizar la prima de una opción americana de venta en términos del premio al riesgo de mercado, el cual es obtenido mediante la solución de una ecuación diferencial parcial lineal de segundo orden. Es importante resaltar, además, que en este planteamiento se ha obtenido la función $g(u)=1-e^{-r u}$ y la fórmula tradicional de Black-Scholes-Merton. La función $g$ se calcula en términos de parámetros conocidos pero (41) requiere de métodos para calcular soluciones aproximadas de $f$. Por supuesto, la tarea de encontrar soluciones aproximadas de $f$ se encuentra ya en una agenda de investigación futura.

La valuación dentro del modelo se lleva a cabo en términos de cuánto estaría dispuesto a pagar el consumidor representativo por mantener un contrato de opción de venta americana optimizando simultáneamente su utilidad por el consumo. Como resultado de este modelo, también se plantea en investigar en el futuro sobre otras formas funcionales de $h\left(\tau_{t}, t\right)$.

Es relevante hacer notar que la principal dificultad de los problemas de control óptimo estocástico reside en resolver la ecuación de HJB, dado que no existe una teoría general para ello. No obstante, para aplicaciones en ciencias económicas, y en particular para el tema de este artículo, es posible encontrar soluciones analíticas y cerradas de dicha ecuación, siempre que se incorpore en los supuestos que la dinámica de los precios es modelada por la ecuación diferencial estocástica del movimiento geométrico browniano y que la función de utilidad es del tipo $U(c, t)=e^{-\rho t} V(c)$, en donde $V$ es un miembro de la familia de funciones de tipo HARA.

Hay que destacar que el argumento que se utiliza en el modelo para evitar la bancarrota, interpretando ésta como la insolvencia del agente económico, es un argumento de tiempo de paro. Este supuesto es sin duda esencial, ya que en 
conjunción con la condición de frontera impuesta a la ecuación de HJB y el uso de una función de utilidad de clase HARA ${ }^{12}$ impiden que el problema degenere $\mathrm{y}$, por ende, evitan la bancarrota del agente económico representativo.

\section{REFERENCIAS BIBLIOGRÁFICAS}

Ángeles, Gerardo, y Venegas-Martínez, Francisco (2010), "Valuación de opciones sobre índices bursátiles y determinación de la estructura de plazos de la tasa de interés de un modelo de equilibrio general", Investigación Económica, 69 (271), pp. 43-80.

Barone-Adesi, Giovanni, y Whaley, Robert (1987), "Efficient Analytic Approximation of American Option Values", Journal of Finance, 42 (2), junio, pp. 301-320.

Black, Fischer, y Scholes, Myron (1973), "The Pricing of Option and Corporate Liabilities”, Journal of Political Economy, 81 (3), mayo-junio, pp. 637-654.

Bjork, Tomas (2004), Arbitrage Theory in Continuous Time, 2a . ed., usA, Oxford University Press.

Bjork, Tomas; Myhrman, Johan, y Persson, Mats (1987), "Optimal consumption with stochastic prices in continuous time", Journal of Applied Probability, 24 (1), pp. 35-47.

Brennan, Michael J., y Schwartz, Eduardo S. (1977), "The Valuation of American Put Options", The Journal of Finance, 32 (2), mayo, pp. 449-462.

Broadie, Mark; Glasserman, Paul, y Ha, Zachary (2000), "Pricing American Options by Simulation Using a Stochastic Mesh with Optimized Weights", en Stanislav P. Uryasev (ed.), Probabilistic Constrained Optimization: Methodology and Applications: Chapter, Dorwell, ma, Kluwer, pp. 26-44.

Broadie, Mark, y Detemple, Jerome (1996), “American option valuation: New bounds, approximations, and a comparison of existing methods", Review of Financial Studies, 9 (4), invierno, pp. 1211-1250.

- (2004), "Option Pricing: Valuation Models and Applications", Management Science, 50 (9), septiembre, pp. 1145-1177.

Clément, Emanuelle; Lamberton, Damien, y Protter, Philip (2002), “An Analysis of a Least Squares Regression Method for American Option Pricing", Finance and Stochastics, 6 (4), pp. 449-471.

\footnotetext{
${ }^{12}$ Esta función de utilidad presenta utilidad marginal infinita cuando el consumo es cero (Merton, 1971; Sethi 1997).
} 
Clower, Robert (1967), "A reconsideration of the microfoundations of monetary theory", Economic Inquiry, 6 (1), diciembre, pp. 1-8.

Cox, John C.; Ingersoll Jr., Jonathan E., y Ross, Stephen A. (1985a). “An Intertemporal General Equilibrium Model of Asset Prices”. Econometrica, 53 (2), marzo, pp. 385-408.

_ (1985b). "A Theory of the Term Structure of Interest Rates". Econometrica, 53 (2), marzo, pp. 363-384.

Cox, John C.; Ross, Stephen A. y Rubinstein, Mark (1979), “Option pricing: A simplified approach", Journal of Financial Economics, 7 (3), septiembre, pp. 229-263.

Cox, John C., y Ross, Stephen A. (1976), "The valuation of options for alternative stochastic processes", Journal of Financial Economics, 3 (1-2), pp. 145-166.

Detemple, Jerome, y Tian, Weidong (2002), "The Valuation of American Options for a Class of Diffusion Processes”, Management Science, 48 (7), julio, pp. 917-937.

Detemple, Jerome; Feng, Shui, y Tian, Wieidong (2003),"The Valuation of American Call Options on the Minimum of Two Dividend-Paying Assets", The Annals of Applied Probability, 13 (3),pp. 953-983.

Duan, Jin-Chuan, y Simonato, Jean-Guy (2001), “American Option Pricing under GARCH by a Markov Chain Approximation", Journal of Economic Dynamics and Control, 25 (11), noviembre, pp. 1689-1718.

Fu, Michael C.; Laprise, Scott B.; Madan, Dilip. B.; Su, Yi, y Wu, Rongwen (2001), "Pricing American Options: A Comparison of Monte Carlo Simulation Approaches", Journal of Computational Finance, 4 (3), primavera, pp. 39-88.

Geske, Robert, y Johnson, Herb (1984), "The American put option valued analytically", The Journal of Finance, 39 (5), diciembre, pp. 1511-1524.

Geske, Robert, y Shastri, Kuldeep (1985), "Valuation by Approximation: A Comparison of Alternative Option Valuation Techniques", The Journal of Financial and Quantitative Analysis, 20 (1), marzo, pp. 45-71.

Grinols, Earl L., y Turnovsky, Stephen J. (1993), "Risk, the Financial Market and Macroeconomic Equilibrium", Journal of Economic Dynamics and Control, 17 (1-2), pp.1-36.

Hakansson, Nils (1970). "Optimal Investment and Consumption Strategies under Risk for a Class of Utility Functions", Econometrica, 38 (5), septiembre, pp. 587-607.

Hernández-Lerma, Onésimo (1994), Lectures on Continuous-Time Markov Control Processes, México, Sociedad Matemática Mexicana.

Heston, Steven L. (1993), "A Closed-Form Solution for Options with Stochastic Volatility with Applications to Bond and Currency Options", Review of Financial Studies, 6 (2), pp. 327-343. 
Ho, Thomas S. Y., y Lee, Sang-Bin (1986), “Term Structure Movements and Pricing Interest Rate Contingent Claims", The Journal of Finance, 41 (5), diciembre, pp. 1011-1029.

Huang, Jing-zhi; Subrahmanyam, Marti G., y Yu., George (1996), "Pricing and hedging American options: a recursive integration method", The Review of Financial Studies, 9 (1), pp. 277-300.

Hull, John, y White, Alan (1987) "The Pricing of Options on Assets with Stochastic Volatilities", The Journal of Finance, 42 (2), junio, pp. 281-300.

- (1993), "One-Factor Interest-Rate Models and the Valuation of Interest-Rate Derivative Securities", The Journal of Financial and Quantitative Analysis, 28 (2), pp. 235-254.

Huyen, Pham (1997), “Optimal stopping, free boundary, and American option in a jumpdiffusion model”, Mathematics and Statistics: Applied Mathematics and Optimization, 35 (2), pp. 145-164.

Ikonen, Samuli, y Toivanen, Jari (2008), "Efficient Numerical Methods for Pricing American Options under Stochastic Volatility", Numerical Methods for Partial Differential Equations, 24 (1), pp. 104-126.

Ju, Nengjiu (1998), "Pricing by American option by approximating its early exercise boundary as a multipiece exponential function", The Review of Financial Studies, 11 (3), pp. 627-646.

Karatzas, Ioannis, y Shreve, Steven (1988), Graduate Text in Mathematics, 113: Brownian Motion and Stochastic Calculus, $2^{\text {a }}$. ed., New York, Springer.

Kohn, Robert (2003), "Partial Differential Equations for Finance", notas de curso [en línea]. Consultado el 30 de enero de 2012, en: http://math.nyu.edu/faculty/kohn/ pde_finance.html.

(2011), “PDE for Finance Notes, Spring 2011-Section 6" [PDF].Consultado el 10 de febrero de 2012 en: http://www.math.nyu.edu/faculty/kohn/pde.finance/ 2011/section6.pdf.

Kou, Steven G., y Wang, Hui (2004), “Option Pricing Under a Double Exponential Jump Diffusion Model”, Management Science, 50 (9), septiembre, pp. 1178-1192.

Longstaff, Francis A., y Schwartz, Eduardo S. (2001), "Valuing American options by simulation: a simple least-squares approach", The Review of Financial Studies, 14 (1), enero, pp. 113-147.

Merton, Robert C. (1971), "Optimum Consumption and Portfolio Rules in a ContinuousTime Model”, Journal of Economic Theory, 3 (4), pp. 373-413.

- (1973), “Theory of Rational Option Pricing”, Bell Journal of Economics, 4 (1), primavera, pp. 141-183. 
_ (1990), Continuous-Time Finance, Cambridge, MA, Basil Blackwell. (1992), “Continuous-Time Finance", Review of Economics and Statistics, 51 (2), pp. 247-257.

Sethi, Suresh P. (1997), Optimal Consumption and Investment with Bankruptcy, USA, Kluwer Academic Publishers.

Shreve, Steven (1997), "Stochastic Calculus and Finance" [PDF]. Consultado el 23 de enero de 2012, en: http://www.stat.berkeley.edu/users/evans/shreve.pdf.

Sierra, Guillermo (2007). "Procesos Hurst y movimientos brownianos fraccionales en mercados fractales", Revista de Administración Finanzas y Economía, 1 (1), pp. 1-21.

Ramsey, F. (1928), “A Mathematical Theory of Saving”, Economic Journal, 38 (152), diciembre, pp. 543-559.

Rogers, Leonard C. G. (2002), "Monte Carlo Valuation of American Options", Mathematical Finance, 12 (3), pp. 271-286.

Stentoft, Lars (2005), "Pricing American Options when the Underlying Asset follows GARCH processes", Journal of Empirical Finance, 12 (4), pp. 576-611.

Taylor, Howard M. (1967), "Evaluating a call option and optimal timing strategy in the stock market", Management Science, 14 (1), pp. 111-120.

Turnovsky, S. J. (1993). "Macroeconomic Policies, Growth, and Welfare in a Stochastic Economy", International Economic Review, 34 (4), pp. 953-981.

Turnovsky, Stephen J., y Smith, William T. (2006), "Equilibrium consumption and precautionary savings in a stochastically growing economy", Journal of Economic Dynamics and Control, 30 (2), febrero, pp. 243-278.

Venegas-Martínez, Francisco (2001), “Opciones cobertura y procesos de difusión con saltos: una aplicación a los títulos de GCARso", Estudios Económicos [en línea], Consultado el 20 de diciembre de 2011, en: http://redalyc.uaemex.mx/redalyc/ src/inicio/ArtPdfRed.jsp?iCve=59716203.

_ (2004), "Reforma fiscal incierta y sus efectos en las decisiones de consumo y portafolio: impacto en el bienestar económico", Problemas del Desarrollo. Revista Latinoamericana de Economía, 35 (136), pp. 137-150.

- (2005), "Política fiscal, estabilización de precios y mercados incompletos", Estudios Económicos, 20 (1), enero-junio, pp. 3-25.

_ (2006). "Stochastic Temporary Stabilization: Undiversifiable Devaluation and Income Risks", Economic Modelling, 23 (1), enero, pp. 157-173.

(2007), "Real Options on Consumption in a Small Open Monetary Economy: A Stochastic Optimal Control Approach", Morfismos, 11 (1), pp.37-52. 
- (2008), Riesgos financieros y económicos, productos derivados y decisiones económicas bajo incertidumbre, $2^{\mathrm{a}}$. ed., México, Cengage.

- (2009), "Un modelo estocástico de equilibrio general para valuar derivados y bonos", Econo Quantum, 6 (1), pp. 111-120.

Venegas-Martínez, Francisco; Ortiz-Arango, Francisco, y Castillo-Ramírez, Claudia E. (2010), "Impacto de la política fiscal en un ambiente con inflación estocástica: un modelo de control óptimo", Morfismos, 14 (1), pp. 51-68.

Venegas-Martínez, Francisco, y Cruz-Ake, Salvador (2010), "Productos derivados sobre bienes de consumo", Econo Quantum, 6 (1), pp. 25-54.

Villeneuve, Stephane (2007), "On Threshold Strategies and the Smooth-Fit Principle for Optimal Stopping Problems”, Journal of Applied Probability, 44 (1), pp. 181-198.

Villeneuve, Stephane, y Zanette, Antonino (2002), "Parabolic ADI Methods for Pricing American Options on Two Stocks", Mathematics and Statistics: Mathematics of Operations Research, 27 (1), febrero, pp. 121-149.

Whaley, Robert E. (1981), "On the Valuation of American Call Options on Stocks with Known Dividends", Journal of Financial Economics, 9 (2), junio, pp. 207-211.

- (1986). Valuation of American Futures Options: Theory and Empirical Tests. The Journal of Finance, Vol. 41, No. 1, marzo de 1986, pp. 127-150.

Wilmott, Paul; Howison, Sam, y Dewynne, Jeff (1999), The Mathematics of Financial Derivatives. A Student Introduction, New York, Cambridge University Press. 